\title{
Palladium-Catalyzed Functionalization of Olefins and Alkynes: From Oxyalkynylation to Tethered Dynamic Kinetic Asymmetric Transformations (DYKAT)
}

\author{
Stefano Nicolai,* Ugo Orcel,* Bastian Muriel,* Phillip D. \\ G. Greenwood,* Luca Buzzetti,* Mikus Purins* and \\ Jerome Waser*a \\ a Laboratory of Catalysis and Organic Synthesis, Ecole \\ Polytechnique Fédérale de Lausanne, EPFL SB ISIC LCSO, BCH \\ 4306, 1015 Lausanne, Switzerland. \\ stefano.nicolai@epfl.ch \\ bastian.muriel@epfl.ch \\ luca.buzzetti@epfl.ch

jerome.waser@epfl.ch

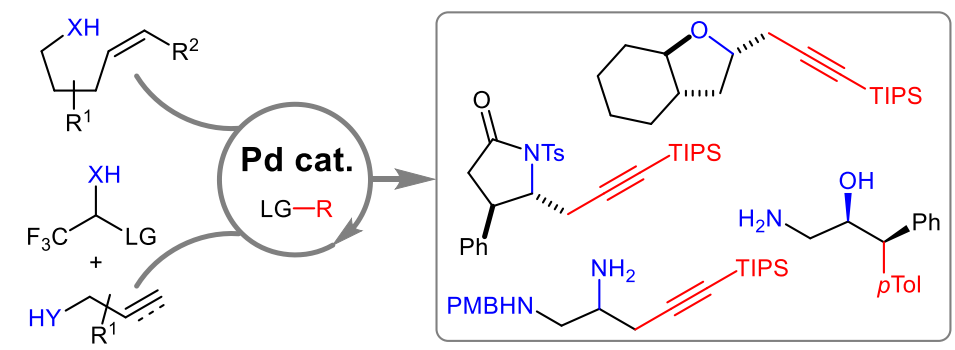

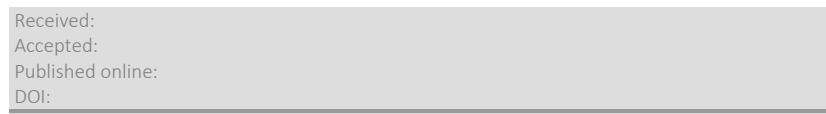

Abstract In this review, an account about the palladium-catalyzed functionalization of alkenes and alkynes developed at the Laboratory of Catalysis and Organic Synthesis (LCSO) is presented. Starting from the intramolecular oxy- and amino-alkynylation of alkenes, tethered methods were then developed to functionalize allylic amines and alcohols, as well as propargylic amines. Finally, a new Dynamic Kinetic Asymmetric Transformation (DYKAT) was developed based on the use of a "one-arm" Trost-type ligand giving access to enantiopure amino alcohols. Each section is a personal account of the researcher(s) who performed the work.

Key words Synthetic methods, Palladium catalysis, alkynes, Trost ligands, DYKAT

\section{Table of Content:}

\section{Introduction}

2. Oxy- and Amino-Alkynylation of Olefins

3. In Situ Tethering Strategies for the Synthesis of Vicinal Amino Alcohols and Diamines

\section{Carboamination of Allylic Alcohols}

5. Carbo-oxygenation of Propargylic Amines

6. Enantioselective Carboetherification/Hydrogenation via a Catalytically-Formed Chiral Auxiliary

\section{Conclusion}

\section{Introduction (Jerome Waser*)}

Palladium catalysts have been extremely successful in synthetic chemistry. ${ }^{1}$ This has been recognized by the awarding of the Chemistry Nobel Prize 2010 to Heck, Negishi and Suzuki for the development of palladium-catalyzed cross-coupling reactions. In 2006, I was a postdoctoral scholar in the Trost group, and even if my personal project involved ruthenium catalysis and total synthesis, palladium catalysis was intensively investigated by other group members. In particular, new asymmetric transformations involving either "triple A" (Asymmetric Allylic
Alkylation) ${ }^{2}$ or "TMM" (trimethylenemethane) ${ }^{3}$ chemistry were flourishing. Using palladium catalysis to develop new transformations in organic synthesis was thus very attractive for me, but I obviously did not want to stay in the same research lines as the Trost group. At that time, I particularly admired the impressive work of the Sanford group on high oxidation state palladium catalysis, often using hypervalent iodine reagents. ${ }^{4}$ I wondered if such reagents could be used to diverge the wellknown palladium-catalyzed Wacker cyclization away from the traditional $\beta$-hydride elimination (path a) process to give more functionalized products (path b) (Scheme 1). A fast oxidation from Pd(II) intermediate I to Pd(IV) complex II, followed by reductive elimination could indeed change the usual reaction pathway. As one of my main research goal was to develop new alkyne chemistry, the use of alkynyliodonium salts appeared especially interesting, considering also the report by Canty and co-workers about stoichiometric oxidative alkynylation of a Pd(II) complex using such reagents. ${ }^{5}$ The model system I draw in my initial research proposal in 2006 was based on a successful Wacker cyclization process reported by Stoltz and co-workers involving phenol as nucleophiles. ${ }^{6}$

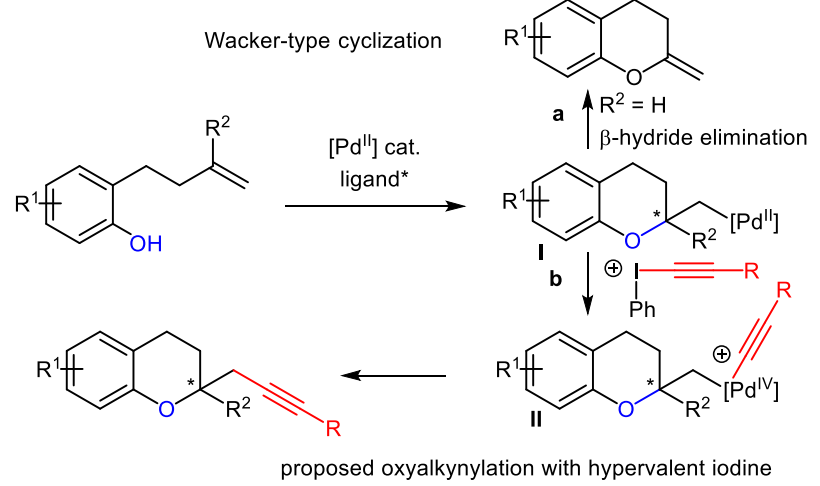

Scheme 1: Original research proposal: from Wacker-type cyclization to oxyalkynylation (10.2006) 
The implementation of this project started in our group in 2008, with the master project of Stéphane Erard. The product could be indeed observed, but only in low yield. It is finally thanks to Stefano Nicolai, who started his PhD in January 2009 that the project could be brought to completion (Scheme 2, eq. 1). ${ }^{7}$ He will tell this story, and also how he could develop in addition an aminoalkynylation ${ }^{8}$ process and a second catalytic system based on $\mathrm{Pd}(0) / \mathrm{Pd}(\mathrm{II})^{9}$ in section 2.

The work of Stefano represented an important breakthrough, but was still based on the intramolecular reaction of a nucleophile with the alkene. When Ugo Orcel joined the group in 2012, I therefore proposed him to develop intermolecular reactions. This was a struggle that was ultimately not successful. Nevertheless, Ugo did not give up and finally developed an in situ tethering methodology for the carbooxygenation and carboamination of allylic amines (Scheme 2, eq. 2). ${ }^{10}$ This general approach was not only useful for alkynylation, but could be also extended to arylation. In section 3, Ugo will explain how he came to this elegant solution.

The approach was not limited to allyl amines. Bastian Muriel could extend the approach to allyl alcohols during his master work under the supervision of Ugo (Scheme 2, eq. 2). ${ }^{11}$ Again, this was not as easy as it could be expected, and new ligands had to be developed (section 4).

When Phillip D. G. Greenwood joined the group in 2015, he received the task to further address the limitations of the tether methodology: in particular, it could be used only for the formation of five-membered ring and with terminal olefins. Overcoming these limitations was important for application of the method in total synthesis. After the usual first year of "fishing", Phill was finally able to extend the method to the carbooxygenation of alkynes (Scheme 2, eq. 3). ${ }^{12}$ In contrast to alkenes, substituents were tolerated and tetrasubstituted olefins were obtained with high stereoselectivity. More about that can be found in section 5 .

Finally, the last chapter of this story was started also by Phill: our first realization of an enantioselective transformation. Indeed, Phill observed that the tetrasubstituted olefins generated by his method could be hydrogenated with complete diastereoselectivity (Scheme 2, eq. 4). It would be "sufficient" to develop an enantioselective access to the oxazolidine to have access to optically pure amino alcohol derivatives. But to realize this goal, an unprecedented palladium-catalyzed Dynamic Kinetic Asymmetric Transformation (a concept well-known in the Trost group) had to be developed. This was extremely challenging and required the work of an exceptional team composed of Phill, Dr. Luca Buzzetti (freshly arrived from the Melchiorre group) and Mikus Purins, a new PhD student and fresh master from Maris Turks group (another former Trost postdoc...) (section 6). After two years of effort, this last project was successful. ${ }^{13}$ Interestingly, the lead result was obtained with the DACH-type Trost ligand, a class of ligands developed for "triple A" reactions, ${ }^{2}$ which has also been used by our group. ${ }^{14}$ However, the best ligand was surprisingly a "one-arm" phosphine truncated derivative $\mathbf{1}$, which had been useless so far in catalysis.

To give a different insight into the story of palladium in our laboratory, I decided to give green card for writing to the group members daily involved in the research, and to design them as corresponding author(s) for the related section. This will give a valuable and different insight into the daily fight and up and down phases in the organic chemistry laboratory.
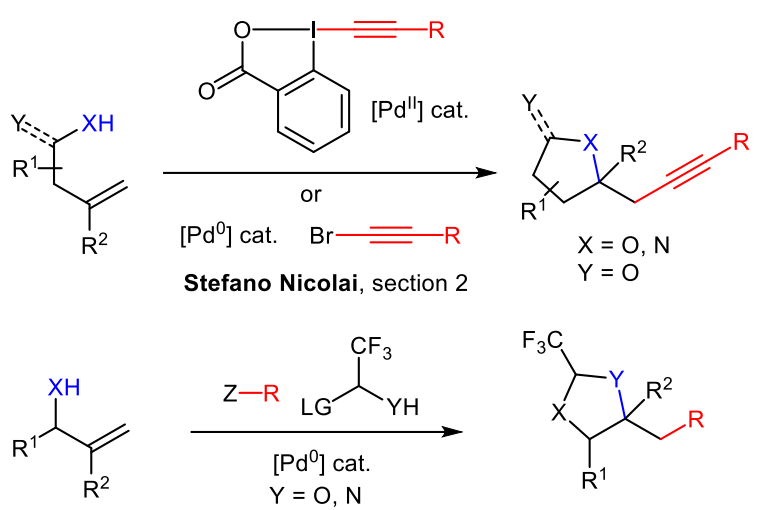

$X=N$, Ugo Orcel, section 3 $\mathrm{X}=\mathrm{O}$, Bastian Muriel, section 4

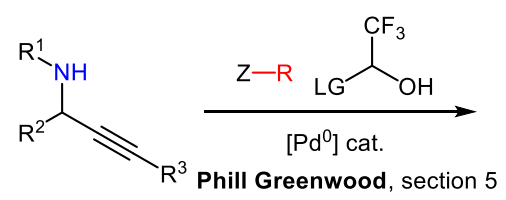<smiles>[R]C([R])=C1OC(C(F)(F)F)N([R])C1[R]</smiles>

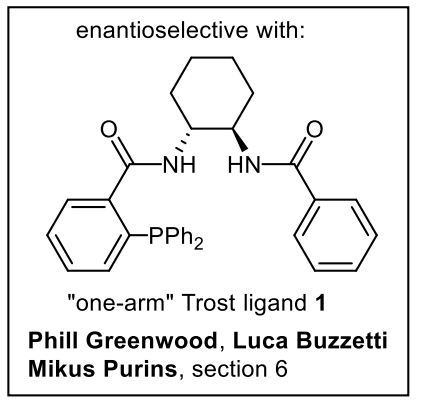

$\mathrm{R}^{2}=\mathrm{H} \downarrow \begin{gathered}\mathrm{Pd}(\mathrm{OH})_{2} \text { cat. } \\ \mathrm{H}_{2}\end{gathered}$<smiles>[R]C([R])[C@H]1CN([R])[C@H](C(F)(F)F)O1</smiles>

Scheme 2: Research in palladium catalyzed functionalization of alkenes and alkynes performed at LCSO from 2009 to 2020.

\section{Oxy- and Amino-Alkynylation of Olefins (Dr. Stefano Nicolai*)}

When I joined Prof. Waser's group in 2009, it was with much enthusiasm that I accepted to start my doctoral research work by investigating the application of palladium catalysis to the intramolecular oxy-alkynylation of olefins. As mentioned in the introduction, Stéphane Erard had been already able to collect encouraging preliminary results by that time. As a proof of concept, he had found that isopropenyl phenol 2a could be cyclized to give propargyl benzo dihydrofuran $4 \mathbf{a}$ in $6 \%$ yield using a palladium salt as the catalyst, together with alkynyl iodonium as electrophilic alkynylating species (Scheme 3). When I took over the project, he had completed a first round of screening, revealing the superiority of the electron-poor complex $\mathrm{Pd}($ hfacac)2, in combination with the neutral hypervalent iodine reagent TIPS-EBX (3). At this point, starting from the highly promising $25 \%$ yield he observed when performing the reaction in methanol, a rapid screening of conditions allowed me to confirm $\operatorname{Pd}(\text { hfacac })_{2}$ as the best catalyst and identify dichloromethane as the optimal solvent. ${ }^{7}$ Substrate $2 \mathbf{a}$ had been initially selected based on the hypothesis that competing $\beta$ hydride elimination might have resulted in the suppression of the desired alkynylation step. We were therefore pleased to find that 
phenol $\mathbf{2 b}$, prone to such a side reaction, was also a competent substrate for the oxy-alkynylation, although leading to a lower $46 \%$ yield of $\mathbf{4 b}$.
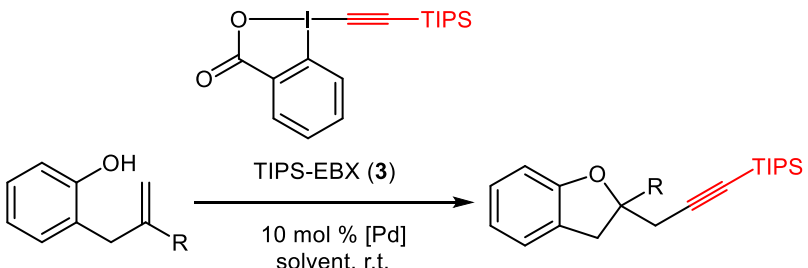

$$
\begin{array}{ccc}
\mathrm{R}=\mathrm{Me},(\mathbf{2 a}) & \mathrm{PdCl}_{2}(\mathrm{MeCN})_{2}, \text { toluene } & \mathrm{R}=\mathrm{Me} \mathrm{(4a)}), 6 \% \\
\mathrm{R}=\mathrm{Me},(\mathbf{2 a}) & \mathrm{Pd}(\text { hfacac })_{2}, \mathrm{MeOH} & \mathrm{R}=\mathrm{Me}(\mathbf{4 a}), 25 \% \\
\mathrm{R}=\mathrm{Me},(\mathbf{2 a}) & \mathrm{Pd}(\mathrm{hfacac})_{2}, \mathrm{DCM} & \mathrm{R}=\mathrm{Me}(\mathbf{4 a}), 73 \% \\
\mathrm{R}=\mathrm{H},(\mathbf{2 b}) & \mathrm{Pd}(\mathrm{hfacac})_{2}, \mathrm{DCM} & \mathrm{R}=\mathrm{H}(\mathbf{4 b}), 46 \%
\end{array}
$$

Scheme 3: Discovery and optimization of the oxy-alkynylation of isopropenyl phenols 2 with TIPS-EBX (3), under Pd(II) catalysis.

The same set of optimized conditions could be then applied to diversely substituted isopropenyl phenols, and then extended to alkenyl benzoic acids, which were satisfyingly converted into propargyl phthalides (5a and $\mathbf{5 b}$ in Scheme 4).

With a broad scope to sustain the value of our new transformation, we were reasonably confident we had secured a high impact publication. The disagreeing opinion of the reviewers prompted us to consider olefin substrates that, so far, had appeared highly unlikely to react under our conditions: aliphatic alcohols and carboxylic acids. While the former remained elusive to the transformation - at least under Pd(II) catalysis (v. infra) - various pentenoic acids were found to undergo the oxy-alkynylation with 70\% yields (for ex. $\mathbf{5 c}$ and $\mathbf{5 d}$ in Scheme 4). Such highly flexible compounds reacted much faster than the previously surveyed ones, demonstrating that: (a) the more acidic character of the aliphatic $\mathrm{CO}_{2} \mathrm{H}$ group makes it more reactive; (b) the entropic gain ensured by the rigid structure of aromatic olefins is not a strict requirement for the success of the cyclization. In addition to this, a most valued result was obtained with unsubstituted pentenoic acid, whose oxyalkynylation occurred in high yield to give $\mathbf{5 d}$ in spite of competitive $\beta$-hydride elimination. This observation had also an important impact when setting the bases of the following project.

Having established a solid protocol for the oxy-alkynylation of alkenyl phenols and carboxylic acids, the development of a "nitrogen variant" of this reaction appeared like an obvious spinoff and an exciting challenge at the beginning of the second year of my PhD. While I was still investigating the scope of the oxyalkynylation, evidence of the viability of a potential aminoalkynylation reaction had been provided under the same conditions by the successful cyclization of benzyl hydroxamate 6a, although poor $\mathrm{N} v s \mathrm{O}$ chemoselectivity was observed with this compound (see products $\mathbf{7 a}$ and 8 in Scheme $5 \mathrm{~A}$ ). ${ }^{7}$ With pentenoic acid now known to be an effective (and a readily available) substrate, I decided to directly address my attention to differently $\mathrm{N}$-activated pentenamides while searching for a model to study the amino-alkynylation. This was the right move: after few unfortunate attempts, easily accessible $\mathrm{N}$-tosyl pentenamide $\mathbf{6 b}$ emerged as the ideal candidate for the investigation of the new reaction. In fact, when I submitted it to reaction with TIPS-EBX (3) in the presence of catalytic
Pd(hfacac) 2 , the desired corresponding propargyl lactam $\mathbf{7 b}$ could be isolated in an encouraging $33 \%$ yield (scheme 5B). ${ }^{8}$

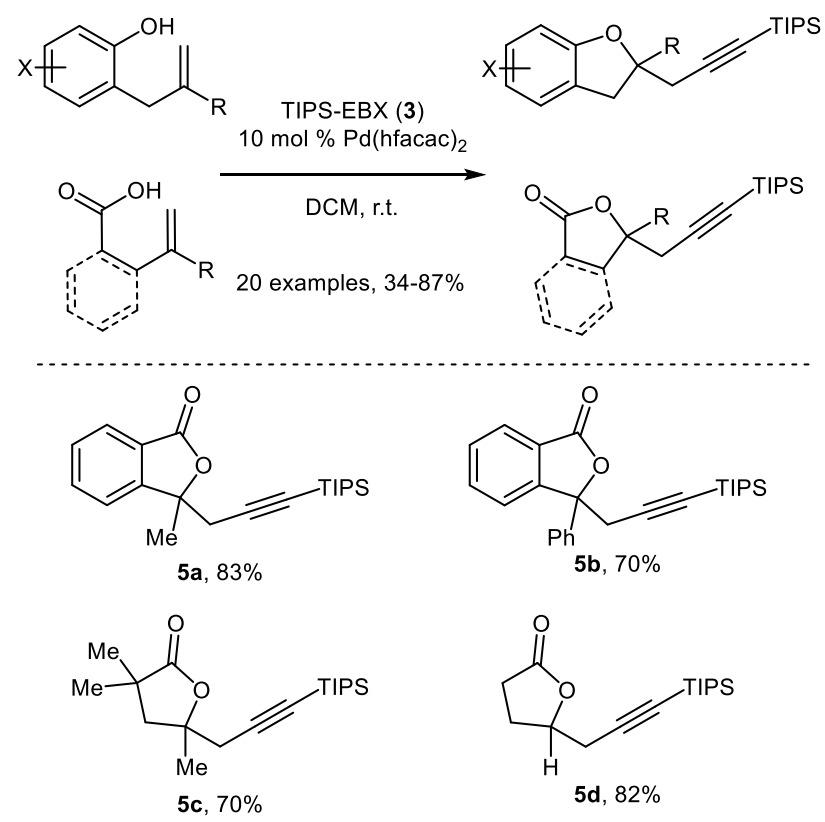

Scheme 4: Selected cope of the oxy-alkynylation reaction.

The hope of another fast optimization was however rapidly disappointed. A first significant yield amelioration up to $57 \%$ was made possible by switching from dichloromethane to chloroform as the solvent, while still using Pd(hfacac)2 as the catalyst. Another improvement came from the survey of several additives, among which ditertbutyl pyridine led to $70 \%$ yield.
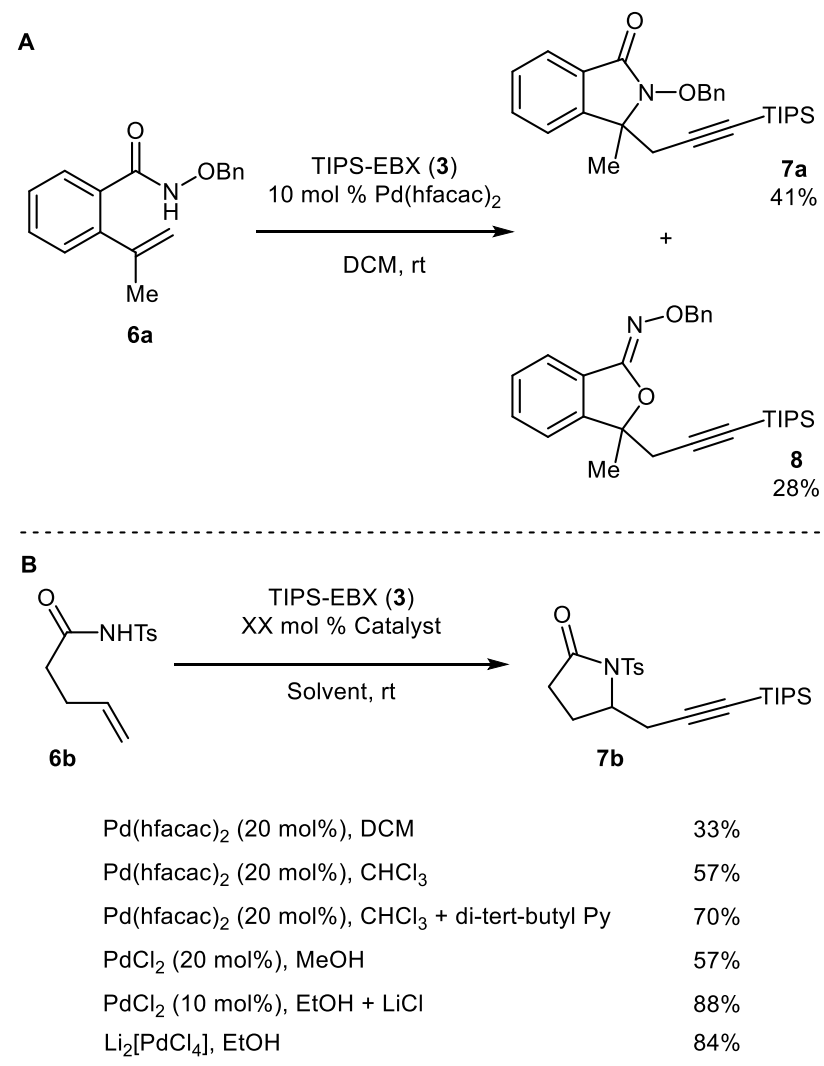

Scheme 5: Discovery and optimization of the amino-alkynylation reaction with TIPS-EBX (3) under Pd(II) catalysis. 
However, it became soon clear that changing the catalytic species had to be envisaged in order to achieve an optimum. In the end, we were please to discover that the simplest and least expensive palladium source - $\mathrm{PdCl}_{2}$ - was the catalyst to choose; the optimal set of (operator friendly) reaction conditions was then found to also include ethanol as the solvent and an excess of additive $\mathrm{LiCl}$, providing the expected propargyl lactam in excellent $88 \%$ yield. A similar result, obtained upon using commercially available $\mathrm{Li}_{2}\left[\mathrm{PdCl}_{4}\right]$, strongly supported the hypothesis that the latter might be the actual active catalytic species, formed is situ under the reaction conditions.

The newly established amino-alkynylation protocol showed to work efficiently with a broad scope of substrates (Scheme 6), giving access to $\gamma$ and $\delta$ lactams (7c-e) in good to excellent yields. The reaction could also be extended to carbamates and ureas $(\mathbf{7 f}$ and $\mathbf{7 g}$ ), which can be seen as early examples of the tethering strategy, later developed within our group. The propargylcontaining heterocycles generated through this method could be then used in an expedient synthetic sequence to access pyrrolizidine and indolizidine scaffold, demonstrating the preparative utility of the amino-alkynylation reaction.

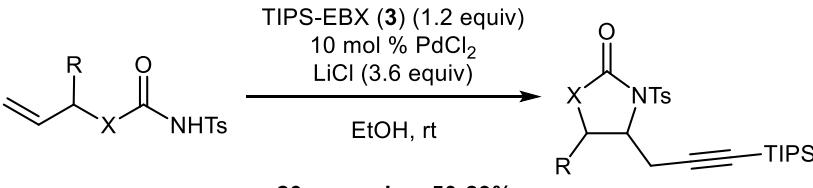

20 examples, $50-89 \%$<smiles>CC1(C)CC(CC#C[In])[N+](=S)C1</smiles>

7c, $84 \%$

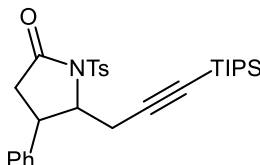

$\mathbf{7 d}, 71 \%(\mathrm{dr}=9: 1)$

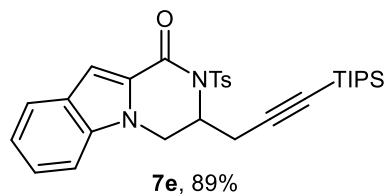

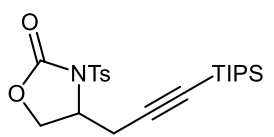

$7 f, 82 \%$

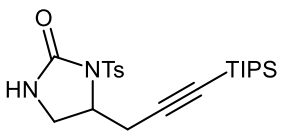

$7 g, 79 \%$
Scheme 6: Scope of the amino-alkynylation reaction with TIPS-EBX (3) under Pd(II) catalysis.

With both oxy- and amino-alkynylation methods now working finely with, respectively, olefin-containing carboxylic acids and $\mathrm{N}$-tosyl amides, for the rest of my $\mathrm{PhD}$, I decided to come back to the problem of less oxidized $\mathrm{O}$ - and $\mathrm{N}$-nucleophiles. Alkenyl alcohols and amines had been found to react sluggishly under the conditions relying on high oxidation state Pd catalytic manifolds and TIPS-EBX (3) as the alkynylating species. The best result I could collect was in fact a less than $20 \%$ yield, obtained in the oxy-alkynylation of pentenol with $\mathrm{Pd}(\mathrm{hfacac})_{2}$. It was at this moment that my interest was caught by the reports of Wolfe and co-workers on $\mathrm{Pd}(0 / \mathrm{II})$ oxy- and amino-arylation reactions. ${ }^{15}$ Based on and similarly to those works, I reasoned that the use of a more electron-rich palladium catalyst would be more compatible with less oxidized nucleophiles. At the same time, less electrophilic alkynylating reagents, like acetylene halides, would be better suitable: this fact was considered as an advantage from the synthetic point of you, considering the rather complex protocol then available for the preparation of EBX reagents (and the still limited variety of the latter). This intuition proved correct: when I first submitted pentenol to reaction with TIPSethynyl bromide (9) in the presence of a catalytic amount of $\mathrm{Pd}_{2}(\mathrm{dba})_{3}$ and DPE-Phos as ligand, I could then isolate the desired propargyl tetrahydrofuran in very good $69 \%$ yield, using THF as the solvent. ${ }^{9 a}$ This very promising result definitively convinced us that it was time to drop Pd(II)/TIPS-EBX (3) and adopt $\mathrm{Pd}(0 / \mathrm{II}) / \mathrm{Br}$-acetylenes as a potentially more rewarding alkynylating system. Optimizing the oxy-alkynylation of pentenol required only minimal effort: although various solvents, palladium sources, and solvents were tested, it sufficed to simply switch from THF to toluene to perform the reaction in excellent 92\% yield (Scheme 7). Under these optimal conditions, the reaction worked efficiently with a broad variety of alkenols. Noteworthy, starting from secondary alcohols, the formation of the corresponding trans-2,5-disubstituted tetrahydrofurans was obtained (10b and 10c). The protocol could also be directly extended to the cyclization of N-protected amines, which could be efficiently converted into the corresponding pyrrolidines $\mathbf{1 0 d}$ and 10e. This is of particular interest, especially when considering that, in palladium catalysis, moving from oxygen to nitrogen nucleophiles generally requires the tedious retuning of most parameters.
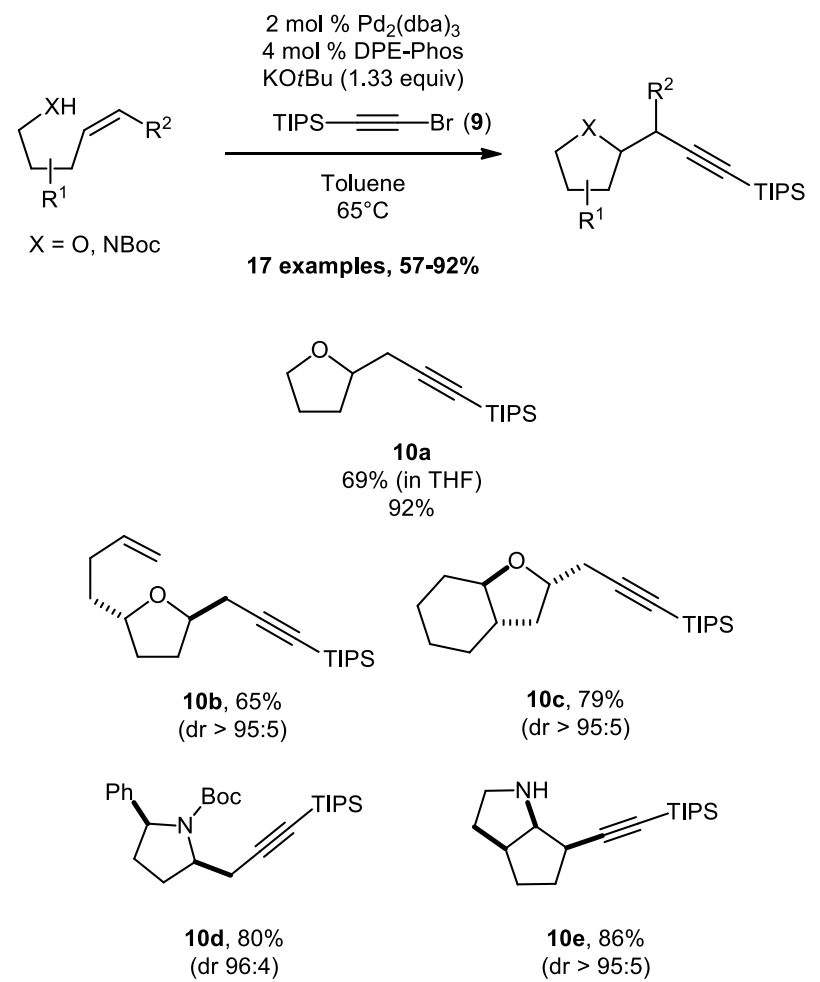

Scheme 7: Oxy- and amino-alkynylation of alkenyl alcohols and amines under $\mathrm{Pd}(0 / \mathrm{II})$ catalysis.

The remaining of my doctoral investigation was essentially focused on the further expansion of the scope of the $\operatorname{Pd}(0 / \mathrm{II})$ catalyzed nucleo-alkynylation of alkenes to aliphatic bromoacetylenes, in alternative to brominated silyl alkynes. ${ }^{9 b}$ The preliminary results I could collect in the carbo-alkynylation 
of alkenyl containing malonates represented instead the starting point for the master's work of Mr. Peter Swallowed, when he joined the group in 2012.9c

\section{In Situ Tethering Strategies for the Synthesis of Vicinal Amino Alcohols and Diamines (Dr. Ugo Orcel*)}

When I started my PhD in 2012, Stefano was already away from the bench and close to finish the writing of his thesis. His work had paved the way for future developments of Pd-catalyzed olefin difunctionalizations in the Waser's group. My goal was initially to extend the scope of oxy- and aminoalkynylation to a fully intermolecular process. After a broad screening of olefin substrates and nucleophiles, a hit was found that involved styrene, alcohols and TIPS-EBX (3). This system had unfortunately strong limitations. One of them was the need of a large excess of olefin and alcohol to obtain a good conversion to the desired oxyalkynylation product. ${ }^{16}$ It was apparent that this system was lacking both reactivity and selectivity, which are typical challenges in multicomponent reactions.

During my investigations, I became aware of the work of the Beauchemin's group on Cope-type hydroamination of allylamines. ${ }^{17}$ Aldehydes are employed to form a mixed aminal insitu which then undergoes a facilitated retro Cope elimination. The singularity of their strategy is the in situ installation and cleavage of the tether that avoids the tedious extra steps usually required to install and remove the tether. That was very intriguing and opened new perspectives for my work. Indeed, applying such strategy by combining an allylamine and an aldehyde tether would form an hemiaminal in situ, whose oxygen would serve as an oxygen source for the oxy-palladation (Scheme 8). This could enable a significant rate acceleration and a higher selectivity for the olefin difunctionalization process by reducing the entropic penalty and by preorganizing the substrate.

The working hypothesis and possible side reactions are depicted in Scheme 8. Firstly, one key to the success of the envisioned reaction is the fast formation of a stable hemiaminal I. Indeed, the allyl amine could undergo undesired Heck or Buchwald-Hartwig cross-coupling reactions in presence of a Pd catalyst, or could deactivate it. Thus, hemiaminal I should be both kinetically and thermodynamically favored over the allyl amine and the iminium II. Acyclic hemiaminals can be stabilized by electronwithdrawing groups on the nitrogen as well as on the ketone or aldehyde partner. ${ }^{18}$ However, the reduced nucleophilicity of the allyl amine could also hamper the formation of the hemiaminal. Secondly, the hemiaminal must also engage in the Pd-catalyzed carbooxygenation process to yield oxazolidines as protected amino alcohols. The putative mechanism starts with the oxidative addition of an organohalide by a $\operatorname{Pd}(0)$ complex III to form the complex IV. Ligand exchange generates the Pd(II)-alkoxyde $\mathbf{V}$. Then, the key oxypalladation occurs to form VI. Alternatively, an undesired $\beta$-hydride elimination could yield an amide. A final reductive elimination step allows the formation of the desired oxazolidine and regenerates the $\operatorname{Pd}(0)$ catalyst.
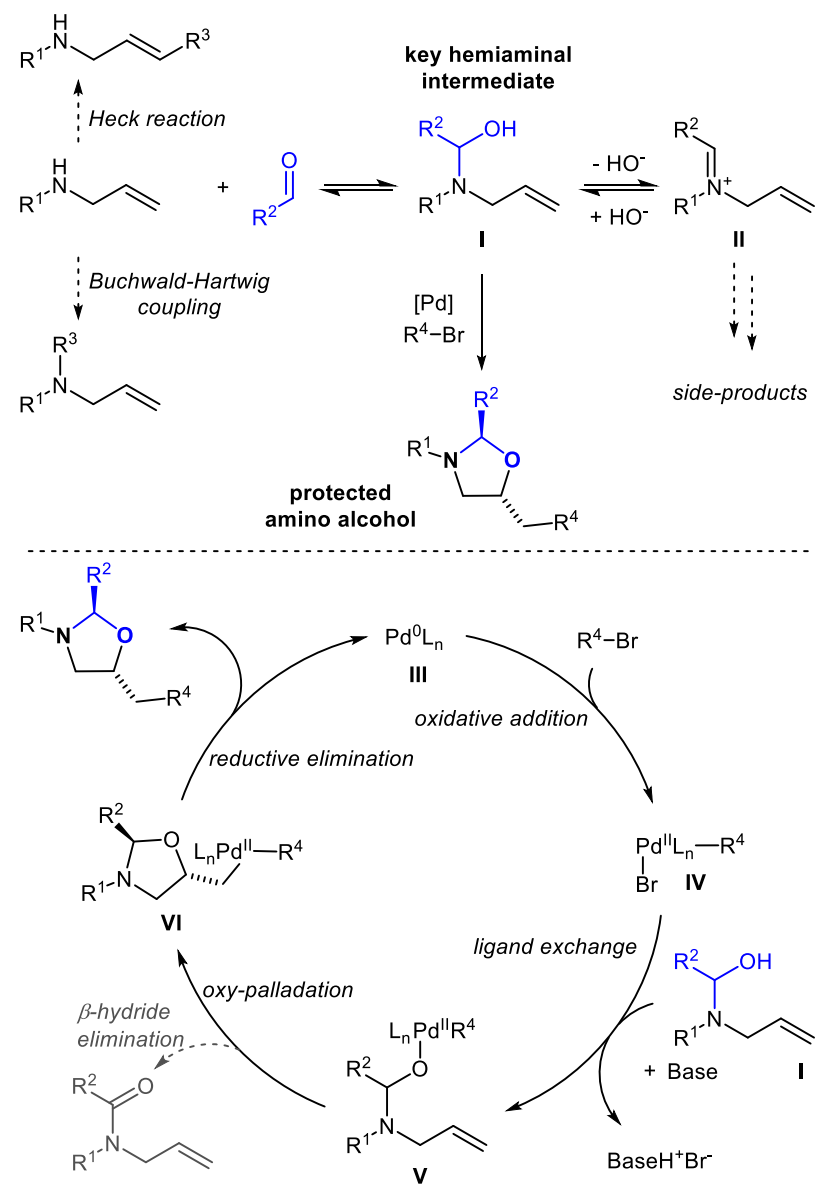

Scheme 8: Concept, challenges and putative mechanism of the tethered carbooxygenation.

To test this approach, I selected (a) two allyl amines with either an electron-withdrawing group (Cbz) or an electron-rich group (Bn), and (b) three aldehydes with different stereoelectronical properties: formaldehyde, benzaldehyde and fluoral under its stable hemiacetal form 11 (Scheme 9). Employing Stefano's $\mathrm{Pd}^{0} / \mathrm{Pd}^{\mathrm{II}}$ conditions gratifyingly allowed me to identify a hit with the combination of the $N$-benzylallyl amine and fluoral ethyl hemiacetal 11, delivering $32 \%$ yield of the desired oxazolidine 12a. Other aldehydes or ketones tested were not superior. The carbonate base, Pd precatalyst and phosphine ligand proved to be critical too. ${ }^{10 a}$ Each class of allyl amine substrates (simple allyl amines (12a-e), $\alpha$-branched allyl amines (12f and 12g), geminally disubstituted olefins $(\mathbf{1 2 h}$ and $\mathbf{1 2} \mathbf{i})$ required a dedicated ligand. The scope of the reaction was broad regarding both the allyl amine and bromide: $\alpha$-branched allyl amines and geminally disubstituted olefins gave the corresponding oxazolidines 12f, 12g and $12 \mathrm{~h}, 12 \mathrm{i}$ in good yields. Electrondeficient aryl and vinyl bromides were also suitable partners in the carbooxygenation process (12j-1). Interestingly, the transformation was often highly diastereoselective, which was a clue that the hemiaminal stereocenter could be epimerized likely via iminium formation - before the oxypalladation would occur. Hence, a DYKAT might be possible with the use of a suitable chiral Pd catalyst. 


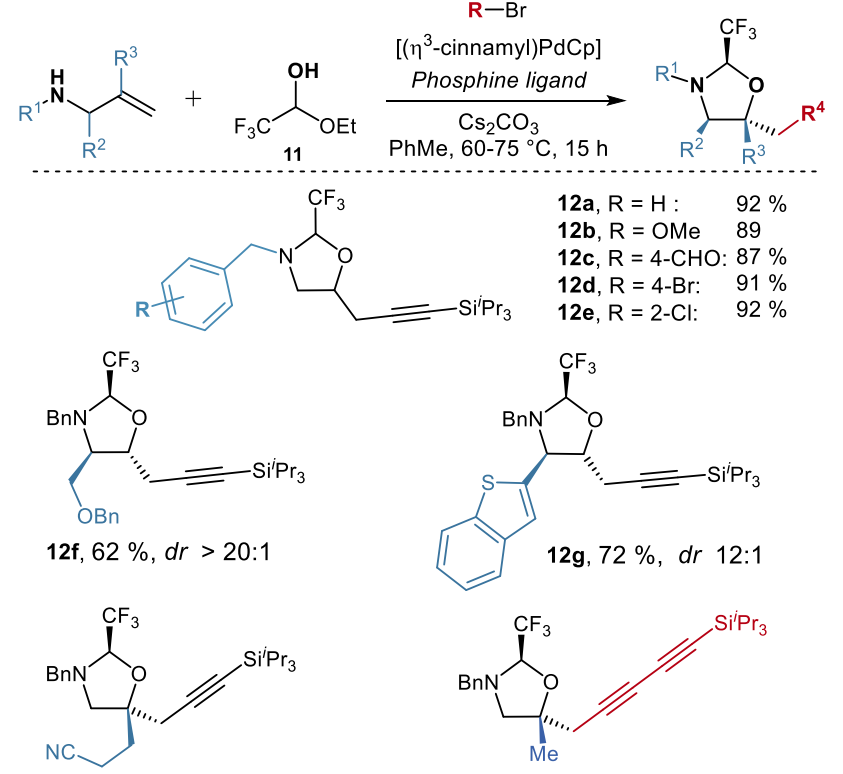

12h, $63 \%, d r>20: 1$
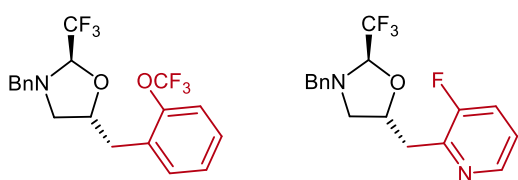

$2 \mathbf{i}, 70 \%, d r 8.3: 1$

12j, $67 \%, d r 2: 1$

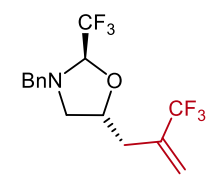

$121,89 \%, d r>20: 1$

Scheme 9: Representative examples of the scope of the carbooxygenation of allyl amines.

The synthetic potential of this methodology was demonstrated with the selective access to: (a) the free amine $\mathbf{1 3}$ by oxidative cleavage of the PMB, (b) the free alcohol 14 by reductive opening of the hemiaminal and (c) the free amino alcohol $\mathbf{1 5}$ by hydrolysis (Scheme 10).

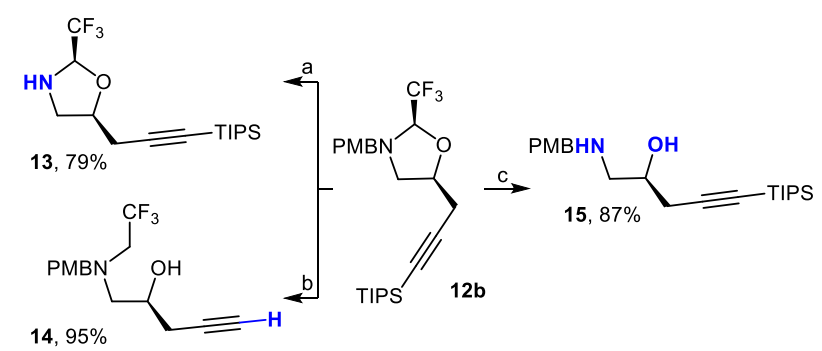

Scheme 10: Orthogonal deprotections of oxazolidines: (a) DDQ, $\mathrm{MeCN}, \mathrm{H}_{2} \mathrm{O}$; (b) DIBAL-H, toluene; (c) PTSA, THF/MeOH, $60^{\circ} \mathrm{C}$.

The logical continuation was the development of a tethered carboamination to access diamines that are extremely valuable building blocks. To this end, an aldimine would be used instead of an aldehyde to form a mixed aminal intermediate, which would then undergo a carboamination to deliver versatile imidazolidines. I initially focused my efforts on identifying a suitable secondary aldimine tether. While the range of aldimines that could deliver the desired imidazolidines was wider than my previous project with aldehydes, only the combination of the $\mathrm{CF}_{3}$ moiety and a carbamate protecting group enabled full conversion and high yields (Scheme 11).10b In contrast to the previous hemiaminal intermediates the mixed aminals were stable enough to be isolated.

The catalytic system involved $\mathrm{Pd}_{2} \mathrm{dba}_{3}$ and mainly $\mathrm{P}(2 \text {-furyl })_{3}$ as ligand. Unlike the synthesis of amino alcohols, this method tolerated both primary and secondary aliphatic allylamines substrates, and also a less nucleophilic aniline derivative (16b). $\alpha$-tertiary amine $16 \mathbf{c}$ could also be accessed provided the use of PhDavePhos as ligand. CsOTf as additive was crucial to obtain high conversion for the more hindered $\alpha$-substituted allylamines such as the complex imidazolidine 16d. CsOTf was also efficient to limit or suppress the undesired Heck reaction when aryl bromides were employed. Thus, a large variety of aryl and hetereoaryls groups could be introduced in high yields, including the pyrimidine $\mathbf{1 6 e}$ and thiophene $\mathbf{1 6 f}$.

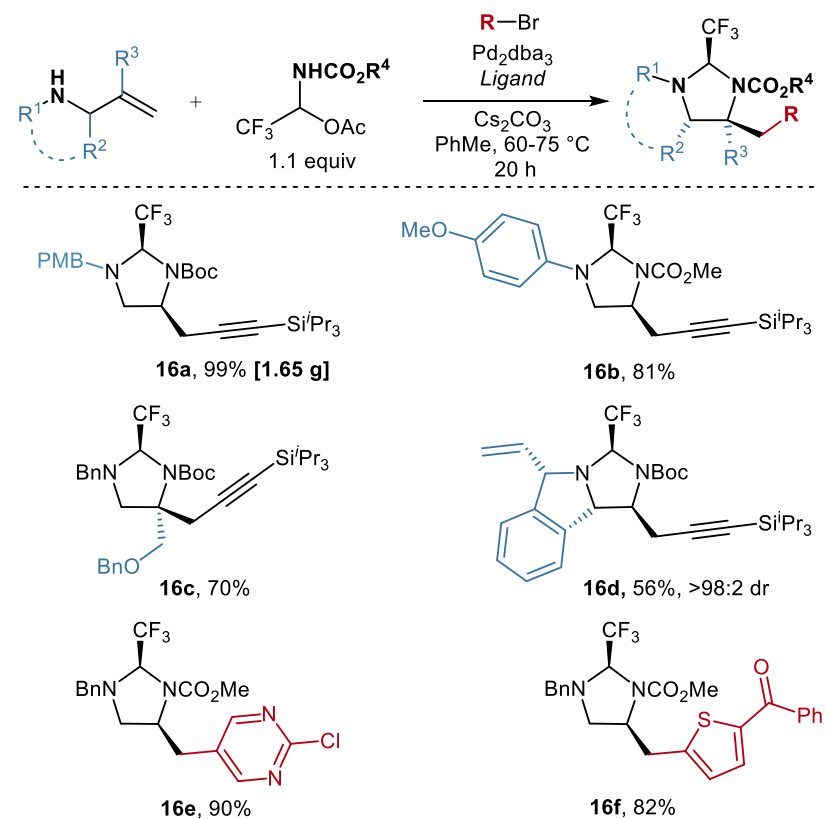

Scheme 11: Representative examples of the scope of the carboamination of allylamines.

The free amines $\mathbf{1 7}$ and $\mathbf{1 8}$ could be accessed selectively depending of the reaction conditions (Scheme 12). Alternatively, full tether removal was achieved by heating in the microwave oven at $160^{\circ} \mathrm{C}$ followed by addition of $\mathrm{HCl}$ in methanol to give 19 .

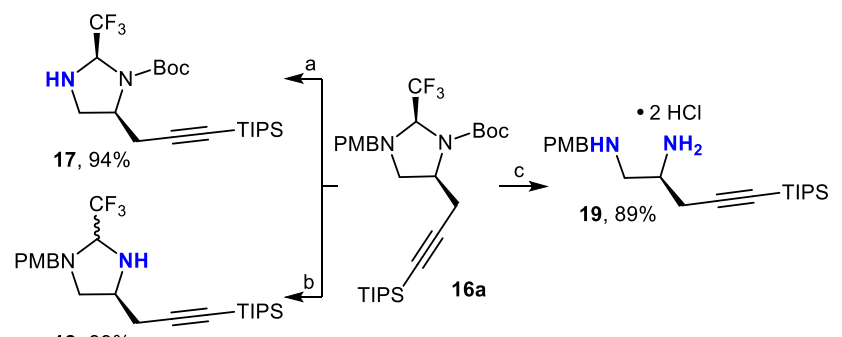

$18,89 \%$

Scheme 12: Orthogonal deprotections of imidazolidines 16a: (a) DDQ, MeCN, $\mathrm{H}_{2} \mathrm{O}$; (b) microwave irradiation $\left(160^{\circ} \mathrm{C}\right), \mathrm{EtOH}: \mathrm{H}_{2} \mathrm{O}$; (c) $\mathrm{HCl}, \mathrm{MeOH}$.

The success of these two methodologies stems largely from the unique effectiveness of $\mathrm{CF}_{3}$-based tethers. Their high electrophilicity and compact size enable: (a) fast and quantitative (hemi)aminal formation in combination with nucleophilic allylamines, and (b) the required stabilization of the resulting (hemi)aminal while retaining sufficient nucleophilicity to undergo the carbooxygenation and carboamination processes. 
Furthermore, the increased acidity of the nuclophiles allow mild carbonate bases to be used and therefore the reactions were tolerant to many functional groups.

\section{Carboamination of Allylic Alcohols (Bastian Muriel*)}

I joined the LCSO group in January 2016 for my master thesis, to investigate new palladium-catalyzed olefin functionalizations under the supervision of Ugo. After several unfruitful investigations towards the carboamination of fluoro-olefins we turned our attentions towards allylic alcohols hoping that we could extend the tethering strategy successfully developed by Ugo with allylic amines. Our interest to develop such a transformation was that it would lead to structurally complementary 1,2-aminoalcohols compared to the ones obtained in Ugo's first project. The first challenge of this work was to find a suitable tether to be introduced on the alcohol, due to its lower nucleophilicity compared to amines. Drawing inspiration from the literature, ${ }^{19}$ we installed different tethers on allyl alcohol $\mathbf{2 0}$ and found that Ugo's tether $\mathbf{2 1}$ derived from trifluoroacetaldehyde allowed for the formation of the corresponding hemiaminal ether $\mathbf{2 2}$ in the highest yield (Scheme 13A). ${ }^{11}$ Moreover, under conditions easily re-optimized from Ugo's last work, the presence of the trifluoromethyl substituent on the tether as well as the Boc-protecting group on the nitrogen were key to attain high yields in the carboamination reaction. While I could achieve the one-pot tether installation / carboamination with allylalcohol 20, the overall yields I obtained remained lower than with allylamines, despite my efforts to optimize this transformation. Hence, after discussions with Professor Waser, we decided to investigate this novel carboamination from the preformed hemiaminal ethers. The second challenge of this project was to find the appropriate phosphine-based ligand to ensure the highest yields and chemoselectivities depending on the substitution on the allylic alcohol as well as the nature of the electrophilic partner (Scheme 13B). Luckily, in combination with bromoalkynes, substituted allylic alcohols delivered the corresponding oxazolidines in high yields and diastereoselectivities in the presence of commercial ligands: XPhos or $\mathrm{P}(2 \text {-furyl })_{3}$. When arylbromides were used however, all the commercial phosphine-based ligands I surveyed led to the formation of a Heck side-product along with the desired aminoarylation. While not optimal in all parts of the scope that Ugo explored for his carboamination of allylic amines, the use of $\mathrm{P}(2 \text {-furyl })_{3}$ often allowed for the formation of the desired products in decent yields. Ugo hence had this idea to introduce furyl substituents on phosphine-based Buchwald-type ligands, hoping that it would be beneficial. He had then synthesized a library of furyl substituted monophopshine ligands that I could test in the aminoarylation I aimed to develop. Gratifyingly, I found that FuXPhos (23) supressed the competing Heck reaction and allowed for the selective formation of the aminoarylation products in good yield. Moreover, I could reach even higher yields by the addition of cesium triflate as additive, an idea previously reported by Wolfe and co-workers, ${ }^{15}$ and also implemented by Ugo. ${ }^{10 \mathrm{~b}}$ Under these conditions a wide range of electron-poor aryl bromides could be successfully engaged in the transformation. Eventually, we could easily remove the trifluoroacetaldehydederived tether from product $\mathbf{2 4}$, to access the corresponding amino alcohol 25 in quantitative yield (Scheme 13C).

A Tether installation

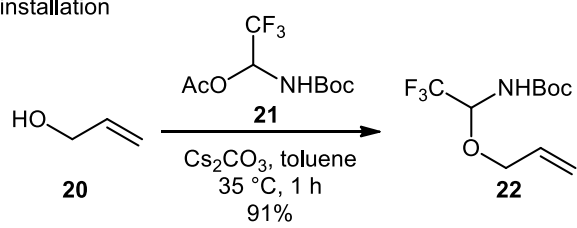

B Optimized conditions for the carboamination and scope

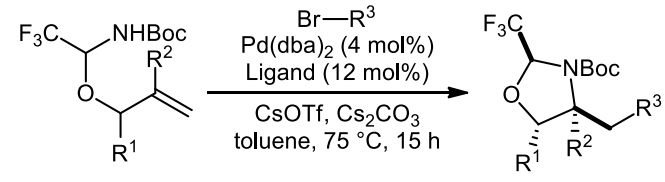

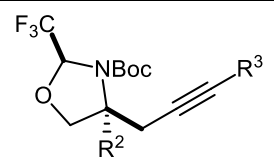

With XPhos

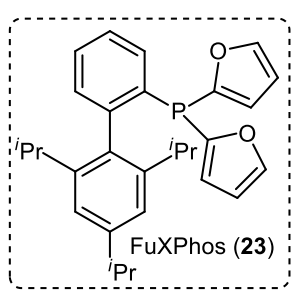

C Tether removal
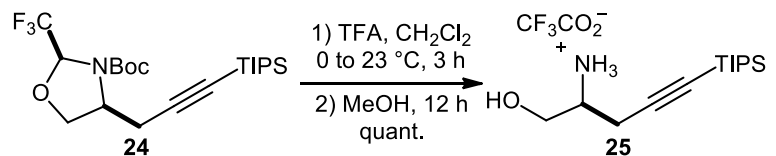

Scheme 13: Carboamination of allylic alcohols bearing a trifluoroacetaldehyde tether.

5. Carbooxygenation of Propargylic Amines (Dr. Phillip D. G. Greenwood*)

I joined the Waser group in October 2015, after spending the last four years in London at Imperial College London and I was immediately taken with the size and space at EPFL. At our meeting after joining the group, Jerome laid out the options of possible projects for me. One of these options seemed like a logical development on the work recently published by the group. This was to develop the tethered carbooxygenation developed by Ugo on the homologated alkene amine system with the desire to access 1,3-amino alcohols (Scheme 14, eq.1). As Ugo had already demonstrated the feasibility of the transformation (with low but promising yields of what was thought to be the desired product) this felt like a good project to start off with for my PhD. After several months of fruitless effort (never exceeding the 13\% NMR yield obtained by Ugo) it was decided that the project would be shelved.

For a second project I wanted to have a go at total synthesis, and I devised a route to a family of tropane alkaloids (Scheme 14, eq. 2) utilising the work of Stefano and Ugo. While there were quite a few steps (12+) to achieve a small and low complexity product, I hoped that it would offer sufficient flexibility and that some of the steps could be removed while showcasing the work done in the group. Unfortunately, while testing a model substrate on the final key reaction involving gold carbene formation from the alkyne developed by Liming Zhang, ${ }^{20}$ the intramolecular cyclisation would preferentially take place on the benzylic 
protecting group rather than the pyrrolidine ring to give $\mathbf{2 6}$ and 27. While other strategies may have been able to provide the desired tropane scaffold, at this point I had lost interest in the project and the synthetic strategy that I had devised.<smiles>[R]CC1([R])OC(C(F)(F)F)N([R])CC1[R]</smiles>

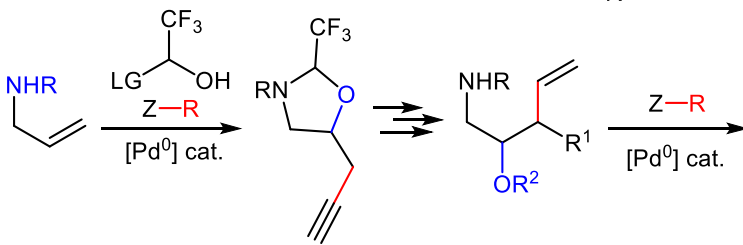

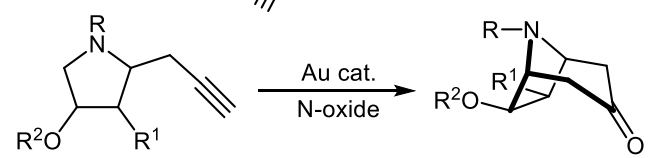<smiles>O=C1CC(c2ccccc2Br)N2CCCCC12</smiles><smiles>Cc1c(CN2CCCC2CC(=O)CC(C)(C)C)cccc1C(C)C</smiles>

Scheme 14: Initial projects and potential applications for the synthesis of tropane alkaloids.

By this point, Ugo had published his paper on carboamination with allylic amines ${ }^{10 b}$ and I went back to thinking of possible ways to develop the chemistry forward. Changing the allylic amine to a propargylic amine looked like it might be an interesting modification (Scheme 15). If the reaction was to proceed then the product formed would contain an additional site of unsaturation in the form of a enol-yne, and if the products were stable it might provide a platform for an array of further product modifications, for example via hydrolysis or hydrogenation.

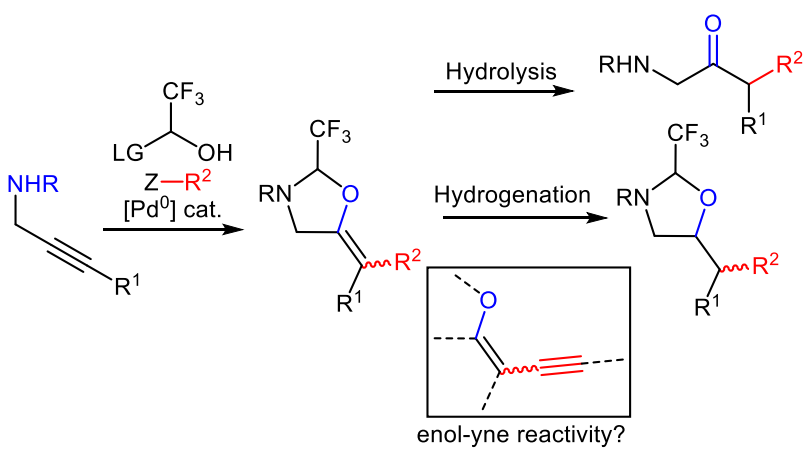

Scheme 15: Carbooxygenation of propargyl amines and potential applications.

One of the selling points of the tether strategy is that the trifluoro acetaldehyde derived tether is cheap and commercially available. The triisopropyl silyl bromoalkyne is easily obtained in one easy step and its purification is essentially a filtration through silica gel. The propargylic amines on the other hand were a pain to make for the next three years with only two of the working starting materials being commercially available (neither of which were model substrates). Several different strategies were used with varying success, with the routes going via tertiary amines leading to the easiest purification. Finally, knowing which of the many reported synthesis protocols really works well for each class of substrates, the starting materials can be made efficiently in a few steps.

I started with the terminal propargylic amine as it provided the closest fit to the successful allylic amines and tested the conditions from Ugo's publication. ${ }^{10 a}$ This immediately supplied us with promising results which after optimisation on the solvent and ligands we were able to improve to give good yields (Scheme 16).12a The screening of conditions quickly showed that a change of solvent to DCE gave an improvement in yield and product selectivity, but changes in the other conditions did not led to any further improvements. The trisubstituted alkene product could be isolated by chromatography but removing all impurities was initially a challenge. It became obvious that the compound was acid sensitive as it would discolour in untreated chloroform and leave a stain on the silica gel if the column was paused for to long, but the product was too apolar to have any triethylamine present. I ended up with a process of treating silica gel in a triethylamine solution, drying the column and running a $0-1 \%$ ethyl acetate in pentane gradient. From the product NMR we saw two product isomers being formed which could be partially separated by preparative TLC, but we had no idea which isomer was which at first. I started on the scope of the different protecting groups hoping that one of them may give a crystal but all the products were oils, it was only a month or so after starting the scope that, looking back through the vials, I noticed one of the products with $\mathrm{N}$-methyl furyl (28) had solidified with some partially formed crystals on the wall, I was unable to perform a recrystallization due to its high solubility but the flecks of crystals that were on the wall were enough for the X-ray crystallography team to analyse and tell us that the major isomer was the Z-isomer indicative of a syn oxy-palladation.
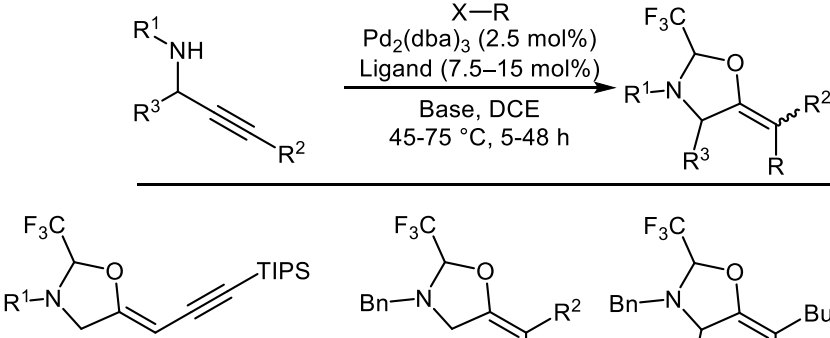

antPhos

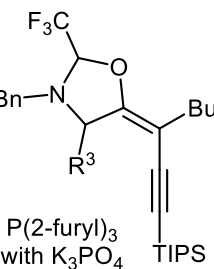

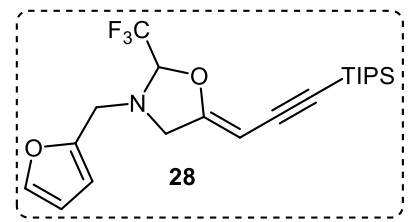<smiles>[R]C(Br)=C1CN(Cc2ccccc2)C(C(F)(F)F)O1</smiles>

RuPhos with $\mathrm{K}_{3} \mathrm{PO}_{4}$

Scheme 16: Carbooxygenation of propargylic amines.

Around the same time as developing conditions for the terminal propargylic substrate, the reaction with the internal propargylic amine 29 was explored (Scheme 17). The success of this substrate would allow access to products with substitution patterns not achievable through the use of allylic amines. Again, the conditions from the allylic amine project immediately gave 
promising results and again, changing the solvent to DCE led to a dramatic improvement in yield for the formation of $\mathbf{3 0}$, but also highlighted a byproduct $\mathbf{3 1}$ being formed. The NMR indicated the byproduct had an increase in planarity and showed peaks from the alkene and alkyne in ${ }^{13} \mathrm{C}$ NMR. The MS showed a mass of the starting material with the addition of TIPS alkyne and $\mathrm{CO}_{2}$ and the IR showed the presence of a $\mathrm{C}=\mathrm{O}$ bond, allowing to assign the structure of 31. But where was $\mathrm{CO}_{2}$ coming from? All reactions were being carried out under $\mathrm{N}_{2}$ and the reactions were degassed quite stringently before being carried out. I was using a carbonate base! As unlikely as it seemed the cesium carbonate was acting as a source of the $\mathrm{CO}_{2}$ unit to form a cyclic carbonate. This was unexpected and interesting but unwanted. The obvious change to make was to use an alternative base in the reaction, one that could not form $\mathrm{CO}_{2}$ in some way. A variety of bases were tried, and potassium phosphate gave the first good hit. I played around a bit at trying to use cesium phosphate, either by simple acid base reaction, or with the use of a furnace. While the furnace produced cesium phosphate provided promising results, analysis determined it to be a mix of di- and tri- basic salt (with the acid base reaction leading to primarily dibasic potassium phosphate). The use of the furnace, while entertaining, was felt to be a bit impractical to implement for a methodology I wish someone else to apply. The initial test with potassium phosphate did give trace amounts of the carbamate, as the bottle used looked rather old, a new bottle was purchased and, as hoped, gave better yield with no carbamate formation. Carrying out some additional optimisation led to an additional change of the ligand to XantPhos (Scheme 16).

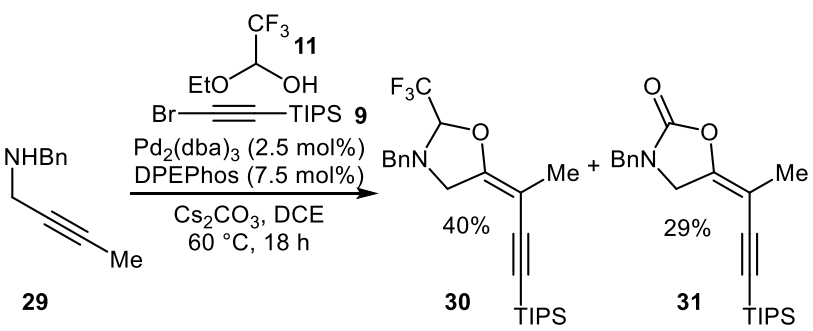

Scheme 17: Carbooxygenation of propargylic amine 29: Unexpected formation of $\mathrm{CO}_{2}$ incorporation side-product 31.

While carrying out the scope of reaction we saw that the E/Z ratio was highly dependent on the substituent of the alkyne, with bulky $t$-butyl or aromatic groups giving exclusively one product. The NMR data indicated that the major isomer was the opposite of the terminal propargylic amines, but we did not have a clear evidence of this as the products were still being obtained as oils. At this point I considered it could be useful to do some derivatisations on product 30, which might be able to provide some crystals for analysis (Scheme 18A). The free alkyne 32, obtained after the removal of the triisopropylsilyl group, still gave an oil. To my surprise and frustration the product $\mathbf{3 3}$ of the copper catalysed 'click' reaction between the free alkyne and parabromo benzylazide still gave the product as an oil. Luckily, I was able to get a reasonable crystal structure from the Sonogashira coupling product 34. The results of the analysis showed that indeed the $\mathrm{E} / \mathrm{Z}$ selectivity had switched indicating a potential change in the mechanism.
A Modification of Alkynylation Product 30<smiles>CC(=C1CN(CC(C)C)C(C(F)(F)F)O1)c1cn(Cc2ccc(Br)cc2)nn1</smiles>

B Modification of Arylation Products

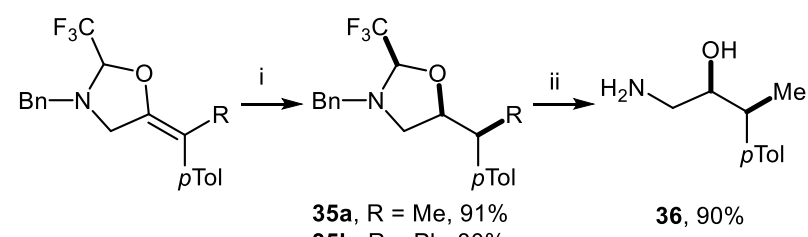

35b, $\mathrm{R}=\mathrm{Ph}, 80 \%$

Scheme 18: Modification of alkynylation and arylation products. A

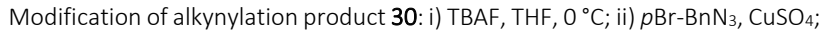
Na-ascorbate, THF: $\mathrm{H}_{2} \mathrm{O}, \mathrm{rt}$; iii) $\mathrm{pNO}_{2}-\mathrm{Ph}-\mathrm{I}, \mathrm{Pd}(\mathrm{OAc})_{2}, \quad$ 1,4diazobicyclo[2.2.2]octane (DABCO), $\mathrm{MeCN}$; and $\mathrm{B}$ Modification of arylation products: i) $\mathrm{H}_{2}, \mathrm{Pd}(\mathrm{OH})_{2} / \mathrm{C}, \mathrm{MeOH}, \mathrm{rt}$; ii) $\mathrm{TsOH}, \mathrm{THF} / \mathrm{H}_{2} \mathrm{O}$, rt.

The addition of substitution at the propargylic position required another screen of conditions for the alkynylation, revealing that now, monodentate tri(2-furyl)phosphine was optimal for the reaction (Scheme 16).

I had the good fortune to work for a short time with Dr. Erwann Grenet, who carried out a large part of the work involving aryl electrophiles and internal propargylic amines. I had made some efforts towards the terminal propargylic amines reacting with aryl electrophiles with limited success. The most useful information obtained was that here, Buchwald type ligands performed better than standard mono- or bidentate phosphine ligands (Scheme 16). Otherwise the reactions led to low yields and mixtures of products. Dr. Grenet was able to develop conditions using aryl iodides and internal propargylic amines, using RuPhos, potassium phosphate and reducing the temperature, leading to good yields as single products. A variety of aryl iodides were successfully submitted with both electron donating and withdrawing groups being tolerated (unlike previous chemistry) and different groups on the propargylic alkyne. The main byproduct seen for these reactions was that of protodemetallation of the vinyl-palladium species. One of the products gave a crystal allowing us to determine that the isomer being formed in the reaction is exclusively from a trans oxypalladation. Dr. Grenet proceeded to carry out the hydrogenation of the obtained arylation products as entries for the product modification (Scheme 18B). The hydrogenation led to an unexpected outcome. We saw that the chosen heterogeneous Pd catalyst gave a single diastereomer of the products $35 \mathbf{a}-\mathbf{b}$ detectable by NMR, which could be easily deprotected to give amino alcohol 36. It is this observation that 
inspired me to pursue an enantioselective transformation at the end of my $\mathrm{PhD}$ (section 6).

Earlier, in the optimisation of the oxyalkynylation with internal propargylic amines I saw that cyclic carbamate $\mathbf{3 1}$ was being formed without the presence of gaseous $\mathrm{CO}_{2}$ as a byproduct (Scheme 17). This was both surprising and interesting as I had not seen a metal carbonate act in such a way with palladium catalysis and thought it might be an interesting reaction to explore further. Other than the work published by Nevado and co-workers, ${ }^{21}$ there were no other palladium-catalyzed cyclic carbamate forming reactions from propargylic amines that proceeded via a dual functionalization of the alkyne. Seeing that the base was playing a key role in the reaction as the source of $\mathrm{CO}_{2}$, this was the starting point for screening. Some screening reactions (with some help from three $1^{\text {st }}$ year masters students at EPFL) of bases and temperature showed that the use of $\mathrm{CsHCO}_{3}$ at $60{ }^{\circ} \mathrm{C}$ would deliver up to $82 \%$ isolated yield of the cyclic carbamates (Scheme 19).12b Two interesting observations that came out of the optimisation: The reaction could be run with a single equivalent of cesium hydrogen carbonate with only a small drop in conversion, and the reaction showed no improvement when additional $\mathrm{CO}_{2}$ was added to the reaction via a balloon. In the publication, we hypothesise a novel mechanism that doesn't involve molecular $\mathrm{CO}_{2 .}{ }^{12 \mathrm{~b}}$ The scope was carried out with a variety of different substituents on the nitrogen or alkyne of the propargylic amine, but unfortunately the attempts to use other electrophile coupling partners were unsuccessful.

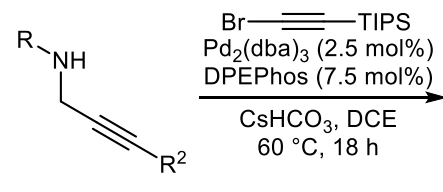

Scheme 19: Carbooxygenation of propargyl amides with $\mathrm{CsHCO}_{3}$ as tether precursor.

6. Enantioselective Carboetherification/Hydrogenation via a Catalytically-Formed Chiral Auxiliary (Dr. Phillip D. G. Greenwood,* Dr. Luca Buzzetti,* Mikus Purins*)

Phill: The seemingly perfect diastereoselectivity of the hydrogenation carried out on the arylation product had been sitting at the back of my mind since we first observed it and I had finished the carboxylation project with six months to go with my $\mathrm{PhD}$. After seeing that the aminoalcohols resulting from the hydrogenation of these products would be pharmaceutically active and that these products would be difficult to obtain enantiomerically by other means, I realized that if I was able to install the $\mathrm{CF}_{3}$ containing tether enantioselectivity, this would open up a route to a series of alkyl-aryl and diaryl amino alcohols. In addition, I had noticed by ${ }^{1} \mathrm{H}$ NMR that, with the propargylic amines, the tether adds reversibly to the amine potentially allowing a dynamic process to take place. After screening a series of chiral mono and bidentate phosphine ligands, ferrocene based JosiPhos ligands with alkyl phosphine demonstrated potential to carry out the carbooxygenation reaction of amine 37 . What proceeded was several months with the invaluable aid of Luca to access a range of ligands based on the JosiPhos scaffold (See Luca's part below for details). Not only were we contending with obtaining good yields and enantioselectivity of the desired product $\mathbf{3 8}$ but also the formation of the protodemetallation product $\mathbf{3 9}$, inseparable by chromatography (Scheme 20). It was only at the suggestion of Jerome, who said it can sometimes be a good idea to test the intermediates of the target ligand as they can also show activity, that we submitted the P,N ligand L1 to the reaction and to my amazement and delight this completely shutdown the protodemetallation product formation and gave the desired product in good yield with a small amount of enantioenrichment. Months were put into variations of the phosphine, amine and side group but a peak was achieved with ligand L2. Some other potential ligand types were tried with limited success. Again, Luca came to save the day by convincing me to try a ligand class that I had missed out on my initial screening, the Trost ligand L3. Immediately the results obtained surpassed the best enantioselectivity obtained thus far with good yield with the only caveat being that the protodemetallation product was being formed again. I tried the other commercial Trost ligands with the of time in the lab and had to start moving to writing my PhD thesis. After handing over my hood and introducing Mikus to the chemistry, I had to take a back seat as he and Luca took it forward.

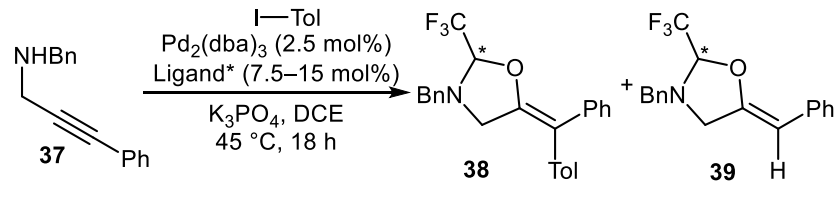

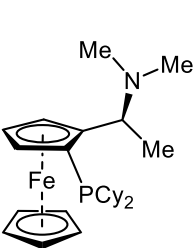

L1

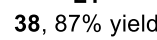

$14 \%$ e.e.

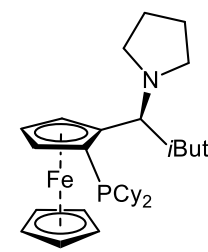

L2
$38,97 \%$ yield

$42 \%$ e.e.

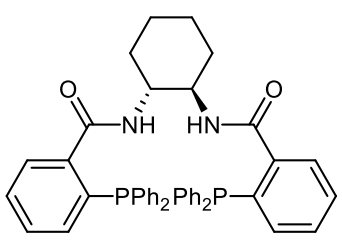

L3 $66 \%$ e.e. $96 \%$ e.e. alternate backbones and side arms but at this point I had run out

$38,66 \%$ yield $\quad 39,29 \%$ yield

Scheme 20: Preliminary results for the enantioselective carbooxygenation.

Luca: I joined the LCSO group in January 2019 as a postdoctoral researcher incorporated in the "tethering funding scheme". This meant I could work on the development of palladium-catalyzed reactions, a field of research that I had never explored before. Since the very first days in the lab, I found particularly intriguing the results obtained by Phill showing a transfer of the stereochemical information during the catalytic hydrogenation of the enantioenriched product 38. Remarkably, the $\mathrm{CF}_{3}$ group, introduced with the tether in an enantioselective fashion, could perfectly control the stereoselectivity of the following reduction: in short, we were exploiting asymmetric catalysis in the carboetherification step to build up a transient chiral auxiliary. At that point the goal was clear: optimize this step both in terms of yield and enantioselectivity to make the whole process efficient and useful: "a few weeks task". Unfortunately, my previous experience in the field of (photo)organo-catalysis was putting me in a slightly less optimistic mood.

Firstly, we started to seek for the best ligand for this dynamic kinetic asymmetric transformation (DYKAT). Our preliminary results relied on the use of the commercially available Josiphos L4, which moderately delivered the desired enantioenriched 
product 38 Scheme 21). However, this catalytic system was mainly yielding the protodemetallation side product $\mathbf{3 9}$, already observed in the previous project. We decided to optimize the ligand structure introducing different phosphines and hoping to improve the e.e. and the product distribution. Although reported, the synthesis of these ligands was time demanding requiring six linear steps from ferrocene (Scheme 21A) and the evaluation of ligands L5-L7 did not bring to any substantial improvement (Scheme 21B, however their column chromatography purification was incredibly artistic). During one of our weekly update, Jerome suggested to directly try the Ugi's amine intermediate $\mathbf{L 1}$ as ligand. Following the golden rule "it's faster to set-up a reaction than to argue" we skeptically try it and for our delight we could observe an almost quantitative formation of the product 38 with complete suppression of the side protodemetallation. The reactivity issue was solved, but we still needed to improve the enantioselectivity. Aiming to achieve this goal, we varied the side alkyl chain of the Ugi's amine ligand (L8L10 $)^{22}$ and after several attempts, we found that an isobutyl group was providing the best results for this class of ligands but we could not improve further the reaction outcome, with only a small improvement with pyrrolidine ligand L2 (Scheme 20).

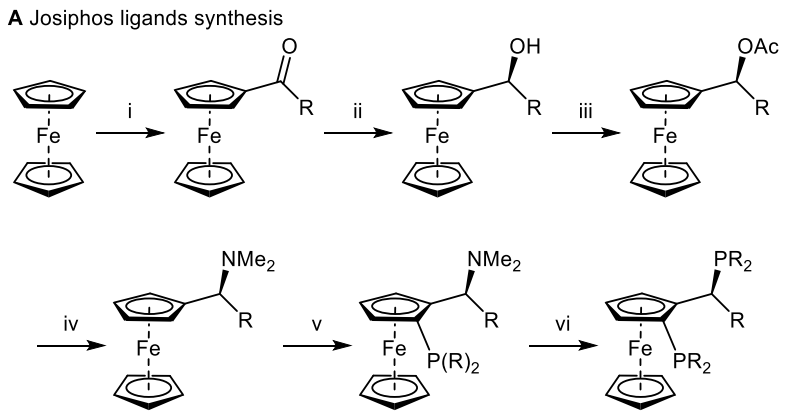

B Results obtained with selected ligands in the carbooxygenation

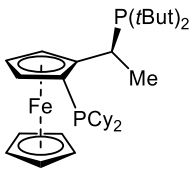

L4

$$
\begin{aligned}
& \text { L4 } \\
& 40 \% \text { yield } \\
& 54 \% \text { e.e. }
\end{aligned}
$$

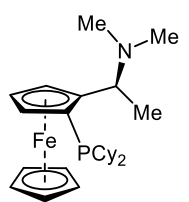

L1

$87 \%$ yield
$14 \%$ e.e.

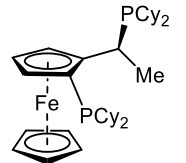

L5

$$
\begin{aligned}
& \text { L5 } \\
& 15 \% \text { yield }
\end{aligned}
$$$$
58 \% \text { e.e. }
$$
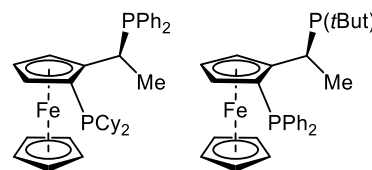

$$
\begin{gathered}
\text { L6 } \\
10 \% \text { yield } \\
28 \% \text { e.e. }
\end{gathered}
$$
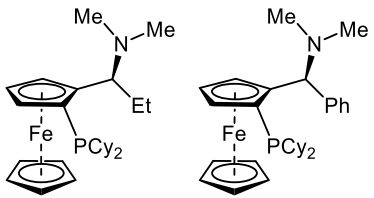

$$
\begin{aligned}
& \text { L8 } \\
& 90 \% \text { yield } \\
& 28 \% \text { e.e. }
\end{aligned}
$$

$\mathbf{L 7}$
$<5 \%$ yield

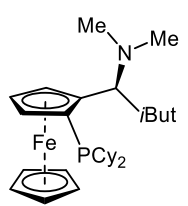

L10

$97 \%$ yield
Scheme 21: A Synthetic route to the Josiphos ligands. i) acylation ii) asymmetric CBS reduction, iii) acetylation, iv) stereoretentive nucleophilic substitution, v) lithiation/phosphorylation, vi) phosphorylation. B Selected results obtained with Josiphos type ligands. Reaction conditions: see Scheme 20.

After closing the ferrocene chapter, we explored other ligand scaffolds. At some point, having an empty space in our heating blocks, we tested an old batch of DACH-Phenyl-Trost L3. We were not expecting any good results out of this trial since the Trost ligands, despite being used in DYKATs, are commonly involved in palladium catalyzed asymmetric allylation, where the palladium complex acts in close proximity to the stereocenter that is forged. ${ }^{2,14}$ Surprisingly, we got the desired product in $66 \%$ yield and $66 \%$ e.e. surpassing all the previously obtained results. Time to start again the "ligand factory" and tune the structure of the Trost ligand to improve the results. Fortunately, these ligands are easily accessible, and their modular synthesis allows the quick preparation of numerous analogs (Scheme 22A). We found a huge batch of monoBoc protected cyclohexyldiamine $\mathbf{4 1}$ prepared (and chirally resolved) by Jerome in 2008 in one of his last adventure in the lab. This served as a precursor for the ligands synthesis. Having in mind the previous positive results with the PN ligands, we substituted the 2 - $\left(\mathrm{PPh}_{2}\right)$-aryl fragment with a 2-pyridine (L11). This modification increased the e.e. to $79 \%$ with a marginal effect on the yield (Scheme 22B). While evaluating the effect of this "second arm" of the ligand, we synthesized the supposed-to-be monodentate $\mathbf{L 1 2}$ and for the third time in few months we got really surprised by the results. This ligand was capable to deliver the desired product in excellent yield and enantioselectivity. We could finally get over $90 \%$ e.e, which is an important goal for the researchers optimizing enantioselective transformations.

A Initial synthetic route towards non-symmetrical Trost-type ligands

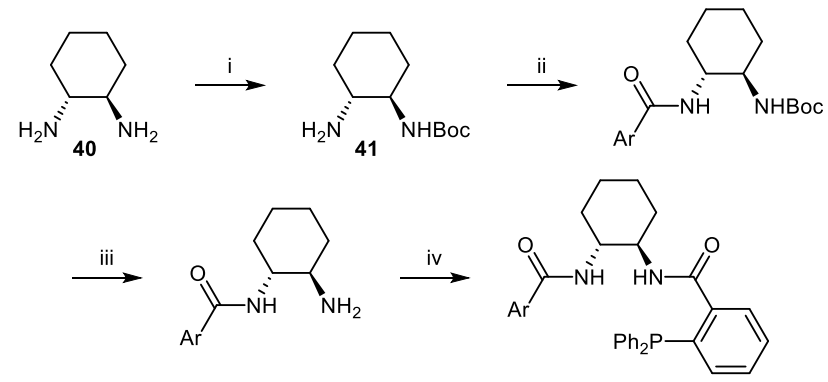

B Results obtained with selected ligands in the carbooxygenation
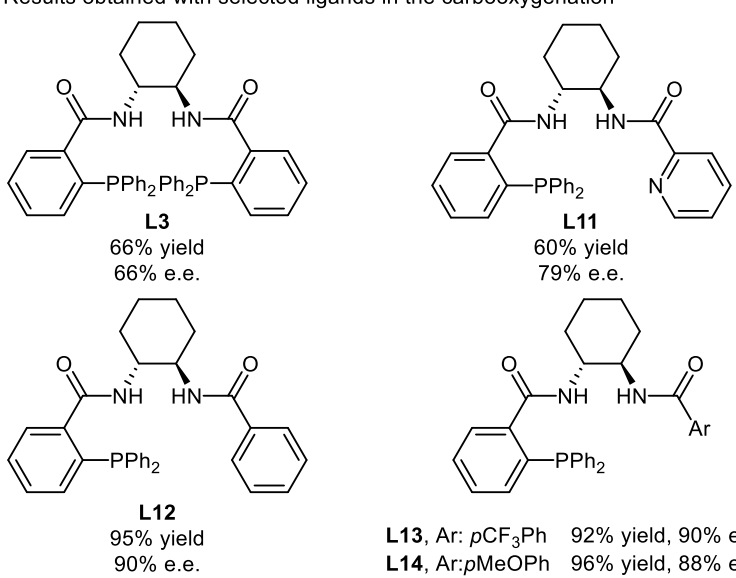

L13, Ar: $p \mathrm{CF}_{3} \mathrm{Ph} \quad 92 \%$ yield, $90 \%$ e.e. L14, Ar:pMeOPh $96 \%$ yield, $88 \%$ e.e.

C Second generation synthesis of ligand L12

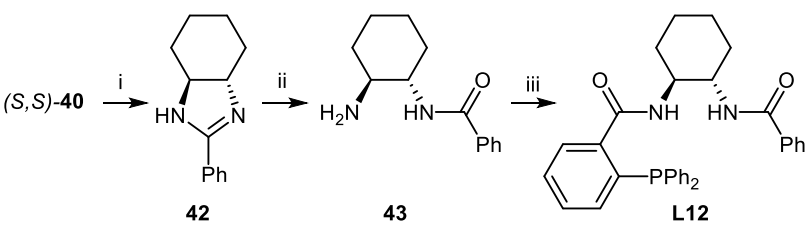

Scheme 22: A Modular synthesis of the "asymmetric" Trost-type ligands. i) Boc protection ( 3 equiv of 40 ), ii) first benzoylation iii) Boc deprotection iv) second benzoylation. B Selected results for the carboetherification with the Trost-type ligands. Reaction conditions: See Scheme 20. C New synthetic route for ligand L12. i) ethyl benzimidate hydrochloride ii) hydrolysis iii) benzoylation. 
Further variation on the ligand structure did not significantly improve the reaction outcome (L13 and L14). For these reasons, we selected $\mathbf{L 1 2}$ as the best ligand for the enantioselective carboetherification and, to obtain a significant amount of it, we developed a cheaper and more practical synthetic route (Scheme 22C). A final tuning of the reaction conditions allowed us to switch the solvent from DCE to diethylether and to reduce the temperature to $35{ }^{\circ} \mathrm{C}$ obtaining the model product of the carboetherification 38 in $>95 \%$ yield and $94 \%$ e.e. on a $0.4 \mathrm{mmol}$ scale. The "few weeks" had turned out to be almost 40 .

Having access to the enantioenriched product $\mathbf{3 8}$ we moved to the optimization of the diastereoselective catalytic hydrogenation. Due to its effectiveness in the reductive cleavage of $\mathrm{C}-\mathrm{N}$ bond, we chose the Pearlman catalyst $\mathrm{Pd}(\mathrm{OH})_{2} / \mathrm{C}^{23}$ aiming to obtain the reduced and deprotected product 39 in a single step. After a short screening of conditions we found a 2:1 mixture of methanol/acetic acid to be the optimal solvent system for avoiding the catalyst poisoning and we obtained the desired product in good yield and without any erosion of the optical purity (Scheme 23). We were finally ready to explore the scope of the process.

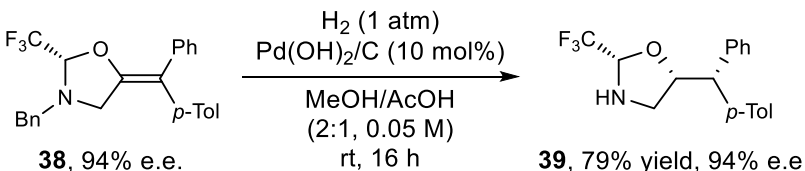

Scheme 23: Optimized condition for the diastereoselective hydrogenation of 38.

Mikus: After fruitful undergraduate research in Riga Technical University, I (M. P.) was up for the challenge of a PhD in organic chemistry. Recommended by Prof. Turks, the Waser group was a good fit with its unconventional approach to synthetic problems. Since my arrival to the LCSO group in September 2019, I had been working together with Luca on the tethered enantioselective carboetherification of propargylic amines. In the initial plan, the project was expected to be finished within $3-4$ months. However, I had already learned the reality of research during my Master's - projects take more time than expected, even if you have already considered this fact. In the first three months, in a control experiment the "one-arm" Trost ligand was found, the fine-tuning of the reaction conditions was finished and the exploration of the scope could be started.

As a neophyte in asymmetric catalysis, at first it was frustrating to set up the same substrate twice - the enantioenriched and the racemic variant. Moreover, due to the nature of a two-step synthetic strategy, the rate of new entries was again cut by a factor of two. Nevertheless, it turned out to be an "easy" scope, with the majority of experiments giving good results (Scheme $24 \mathrm{~A}$ ). We were more concerned about the second step, as we did not know at the time whether the stereoselective hydrogenation would be general. Fortunately, we always observed a single diastereoisomer of reduced products (Scheme 24B). At this point the COVID-19 pandemic situation in Switzerland and globally was worsening by the hour. With the end of lab work within reach (expected approx. 1 month left), EPFL went into full lockdown. The last day at the lab was particularly grim - Luca suggested preparing drinking water reserves, in case the doomsday clock hits midnight. After 6 weeks of complete isolation, we returned part-time to the lab with unprecedented efficiency at the bench due to the limited working-time. Finally, after 6 more weeks at partial occupation, things could go back to the new "normal" and we could finish all of the experiments required for the project.

A<smiles>[R]C#CCN[R]</smiles>

$$
\begin{aligned}
& \mathrm{R}^{1}=\mathrm{Bn}, \mathrm{Ar}, \text { Alk } \\
& \mathrm{R}^{2}=\mathrm{Ar}, \text { Alk } \\
& \mathrm{Z}-\mathrm{R}=\text { (Het)Arl }
\end{aligned}
$$

31 examples up to $99 \%$ yield, $94 \%$ e. e.
Tolerated: EDG, EWG in para position; meta and (ortho) substitution Not tolerated: Sterically demanding substitution in ortho position

B

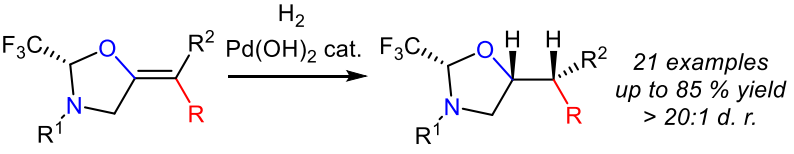

$$
\begin{aligned}
& \mathrm{R}^{1}=\mathrm{H} \text { or Alk, Ar }
\end{aligned}
$$

Tolerated: EDG, EWG in para position; meta and (ortho) substitution Not tolerated: C-Halogen bonds, extra heteroatoms

Scheme 24: Scope of the tethered enantioselective carboetherification of propargylic amines

The mechanism and the model for asymmetric induction of the enantioselective carboetherification reaction remains highly speculative (Scheme 25). We know by NMR experiments that there exists an equilibrium between the propargylic amine/ hemiacetal $\mathbf{1 1}$ and the hemiaminal I. The constant racemization of hemiaminal I combined with a discrimination between the two enantiomers by a chiral Pd catalyst enables a DYKAT. However, the exact missing pieces of the puzzle are unknown, as was correctly pointed out by the committee of my candidacy exam.<smiles>OC(N(CC#C[Al])Cc1ccccc1)C(F)(F)F</smiles>

(S)-I

(S)

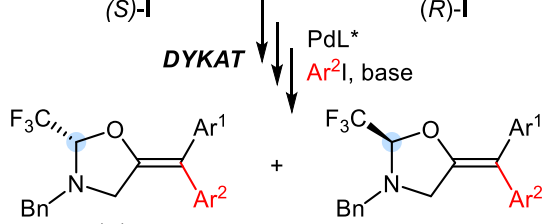<smiles>CCOC(O)C(F)(F)F</smiles><smiles>OC(N(CC#C[Al])Cc1ccccc1)C(F)(F)F</smiles>

$(R)-\mathbf{I}$
$(R)$

Scheme 25: Equilibrium at the basis of the dynamic kinetic asymmetric transformation (DYKAT).

We found the stereoselectivity of the hydrogenation particularly fascinating. The single crystal X-ray structures of oxazolidine $\mathbf{3 8}$ and the reduced oxazolidine 39 (Scheme 26) confirmed both the retention of the absolute configuration, and the approach of hydrogen from the opposite side of the $\mathrm{CF}_{3}$-group. This was relieving, as the contrary would not be the first time in organic chemistry, when the initial hypothesis would be later found to be far from reality. 


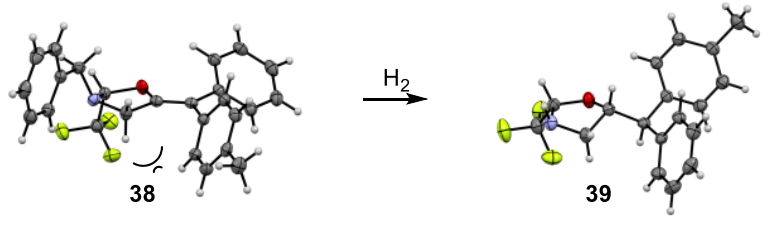

Scheme 26: Rationalization of the side-selectivity in the hydrogenation of $\mathbf{3 8}$ based on its crystal structure.

In fact, the installed $\mathrm{CF}_{3}$-group on the oxazolidine $\mathbf{3 8}$ was acting as a de facto chiral auxiliary. To the best of our knowledge, the concept of a catalytically formed chiral auxiliary starting from non-chiral starting materials had never been reported in the literature. We feel our approach address many issues associated with the use of chiral auxiliaries, and more developments are expected in the future.

\section{Conclusions (Jerome Waser*)}

It has been a privileged for me to work with three generations of talented young scientist on palladium catalysis. The fourth generation just entered the game and I am sure that further exciting results can be expected. The surprise for me was perhaps that part of the initial project based on hypervalent iodine chemistry for oxyalkynylation could indeed be realized, even if no enantioselective methods could be developed at this time. It is always beautiful to see design become reality. This project brought many satisfactions, but also had its lot of frustrations and tough decisions to take. We had to abandon our "beloved" hypervalent iodine reagents when they reached their limits. We couldn't develop general intermolecular transformations, but this forced up to adapt and develop our new tether methodology, which led finally to success in enantioselective catalysis 12 years after the start of the project. At the beginning, there was some design, but the final success came out of pure serendipity, a control experiment that led to the discovery of the truncated Trost ligand L12. Success has outpaced understanding, and it will be highly interesting to investigate the mechanism and mode of stereoinduction for this new DYKAT process. Progress is now in the hand of the next generation of researchers in the lab, and nobody can tell where the future will bring us!

\section{Acknowledgment}

We thank the Swiss National Science Foundation (Grant numbers 20021_119810 and 20021_15992), ERC (European Research Council, Starting Grant iTools4MC, number 334840 and Consolidator Grant, SeleCHEM, number 771170) and EPFL for financial support.

\section{References}

(1) Tsuji, J., Palladium Reagents and Catalysts. John Wiley \& Sons: Chichester, 2004.

(2) (a) Trost, B. M. Acc. Chem. Res. 1996, 29, 355. (b) Trost, B. M.; Bunt, R. C.; Lemoine, R. C.; Calkins, T. L.J. Am. Chem. Soc. 2000, 122, 5968. (c) Trost, B. M. J. Org. Chem. 2004, 69, 5813. (d) Trost, B. M.; Xu, J. Y. J. Am. Chem. Soc. 2005, 127, 2846. (e) Trost, B. M.; Machacek, M. R.; Aponick, A. Acc. Chem. Res. 2006, 39, 747. (f) Trost, B. M.; Quancard, J. J. Am. Chem. Soc. 2006, 128, 6314.
(3) (a) Trost, B. M.; King, S. A. J. Am. Chem. Soc. 1990, 112, 408. (b) Trost, B. M.; Cramer, N.; Bernsmann, H. J. Am. Chem. Soc. 2007, 129, 3086. (c) Trost, B. M.; Cramer, N.; Silverman, S. M. J. Am. Chem. Soc. 2007, 129, 12396. (d) Trost, B. M.; McDougall, P. J.; Hartmann, O.; Wathen, P. T. J. Am. Chem. Soc. 2008, 130, 14960. (e) Trost, B. M.; Bringley, D. A.; Silverman, S. M. J. Am. Chem. Soc. 2011, 133, 7664. (f) Trost, B. M.; Mata, G. Acc. Chem. Res. 2020, 53, 1293.

(4) (a) Desai, L. V.; Hull, K. L.; Sanford, M. S. J. Am. Chem. Soc. 2004, 126, 9542. (b) Dick, A. R.; Hull, K. L.; Sanford, M. S. J. Am. Chem. Soc. 2004, 126, 2300. (c) Dick, A. R.; Kampf, J. W.; Sanford, M. S. J. Am. Chem. Soc. 2005, 127, 12790. (d) Kalyani, D.; Deprez, N. R.; Desai, L. V.; Sanford, M. S. J. Am. Chem. Soc. 2005, 127, 7330. (e) Deprez, N. R.; Kalyani, D.; Krause, A.; Sanford, M. S. J. Am. Chem. Soc. 2006, 128, 4972.

(5) Canty, A. J.; Rodemann, T.; Skelton, B. W.; White, A. H. Organometallics 2006, 25, 3996.

(6) Trend, R. M.; Ramtohul, Y. K.; Stoltz, B. M. J. Am. Chem. Soc. 2005, 127, 17778.

(7) Nicolai, S.; Erard, S.; Fernandez Gonzalez, D.; Waser, J. Org. Lett. 2010, 12, 384.

(8) Nicolai, S.; Piemontesi, C.; Waser, J. Angew. Chem., Int. Ed. 2011, 50, 4680 .

(9) (a) Nicolai, S.; Waser, J. Org. Lett. 2011, 13, 6324. (b) Nicolai, S.; Sedigh-Zadeh, R.; Waser, J. J. Org. Chem 2013, 78, 3783. (c) Nicolai, S.; Swallow, P.; Waser, J. Tetrahedron 2015, 71, 5959.

(10) (a) Orcel, U.; Waser, J. Angew. Chem., Int. Ed. 2015, 54, 5250. (b) Orcel, U.; Waser, J. Angew. Chem., Int. Ed. 2016, 55, 12881. (c) Orcel, U.; Waser, J. Chem. Sci. 2017, 8, 32.

(11) Muriel, B.; Orcel, U.; Waser, J. Org. Lett. 2017, 19, 3548.

(12) (a) Greenwood, P. D. G.; Grenet, E.; Waser, J. Chem. Eur. J. 2019, 25, 3010. (b) Greenwood, P. D. G.; Waser, J. Eur. J. Org. Chem. 2019, $2019,5183$.

(13) Buzzetti, L.; Purins, M.; Greenwood, P. D. G.; Waser, J. J. Am. Chem. Soc. 2020, 142, 17334 ..

(14) (a) Vita, M. V.; Mieville, P.; Waser, J. Org. Lett. 2014, 16, 5768. (b) Vita, M. V.; Caramenti, P.; Waser, J. Org. Lett. 2015, 17, 5832.

(15) (a) Lira, R.; Wolfe, J. P. J. Am. Chem. Soc. 2004, 126, 13906. (b) Ney, J. E.; Wolfe, J. P. Angew. Chem., Int. Ed. 2004, 43, 3605. (c) Wolfe, J. P.; Rossi, M. A. J. Am. Chem. Soc. 2004, 126, 1620. (d) Mai, D. N.; Wolfe, J. P. J. Am. Chem. Soc. 2010, 132, 12157. (e) Neukom, J. D.; Perch, N. S.; Wolfe, J. P. J. Am. Chem. Soc. 2010, 132, 6276.

(16) Unpublished results.

(17) (a) MacDonald, M. J.; Schipper, D. J.; Ng, P. J.; Moran, J.; Beauchemin, A. M. J. Am. Chem. Soc. 2011, 133, 20100. (b) Guimond, N.; MacDonald, M. J.; Lemieux, V.; Beauchemin, A. M. J. Am. Chem. Soc. 2012, 134, 16571.

(18) Van Benthem, R. A. T. M.; Hiemstra, H.; Speckamp W. N. J. Org. Chem. 1992, 57, 6083, and references cited therein.

(19) Weinstein, A. B.; Schuman, D. P.; Tan, Z. X.; Stahl, S. S. Angew. Chem. Int. Ed. 2013, 52, 11867.

(20) Cui, L.; Peng, Y.; Zhang, L. J. Am. Chem. Soc. 2009, 131, 8394.

(21) García-Domínguez, P.; Fehr, L.; Rusconi, G.; Nevado, C. Chem. Sci. 2016, 7, 3914.

(22) Oost, R.; Rong, J.; Minnaard, A. J.; Harutyunyan, S. R., Synthesis of New Derivatives of Copper Complexes of Josiphos Family Ligands for Applications in Asymmetric Catalysis. Catal. Sci. Technol. 2014, 4, 1997-2005.

(23) Pearlman's Catalyst. in Comprehensive Organic Name Reactions and Reagents (John Wiley \& Sons, Inc., 2010). doi:10.1002/9780470638859.conrr483. 


\section{Biosketches}

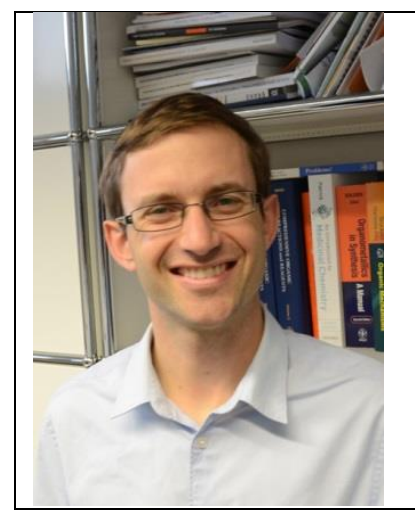

Jérôme Waser was born in Sierre, Valais, Switzerland in 1977. He obtained is chemistry Diploma at ETH Zurich in 2001. From 2002 to 2006, he was a PhD student at ETH Zurich with Prof. Erick M. Carreira. He then joined Prof. Barry M. Trost at Stanford University as a SNF postdoctoral fellow. From 2007 to 2014, he was an assistant professor at EPF Lausanne (EPFL). Since June 2014, he has been associate professor at EPFL. He is a recipient of the ERC Starting Grant 2013 and Consolidator Grant 2017, the Werner prize of the Swiss Chemical Society 2014 and the Springer Heterocyclic Chemistry Award 2016.

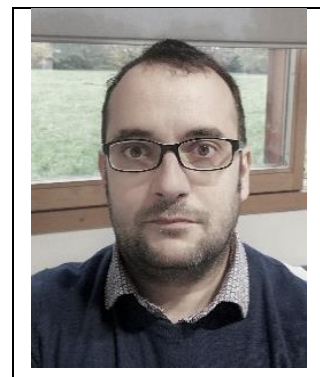

Stefano Nicolai was born in Civitavecchia (metropolitan area of Rome), Italy in 1979. He obtained his Bachelor's and Master's Degrees at the Sapienza, University of Rome, in 2005 and 2007, respectively. From 2009 to 2013, he was a PhD student at the Ecole Polytechnique Fédérale de Lausanne, under the supersion of Prof. Jérôme Waser. From 2014 to 2015, he then worked within the New Synthetic Ingredients Discovery division at Firmenich (Geneva), as a postdoctoral scientist. He joined back Prof. Waser's research group at the EPFL in late 2015, where he is currently working as Associé à la Recherche (Research scientist).

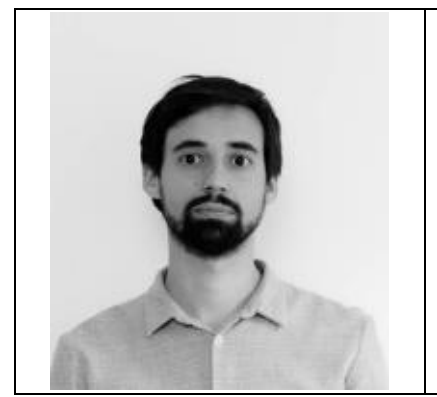

Ugo Orcel was born in Annecy (France) in 1989. He graduated from the Ecole Nationale Supérieure de Chimie de Montpellier (France) in 2012. He completed his PhD at the Ecole Polytechnique Fédérale de Lausanne (Switzerland) in 2017, under the supervision of Prof. Jérôme Waser. He then joined the group of Prof. Bernhard Breit at the Albert-Ludwigs-Universität Freiburg (Germany) to pursue his postdoctoral studies with an Early Postdoc. Mobility fellowship of the SNSF. Since 2018 he is working for F. Hoffmann-La Roche in Basel (Switzerland) in process chemistry.

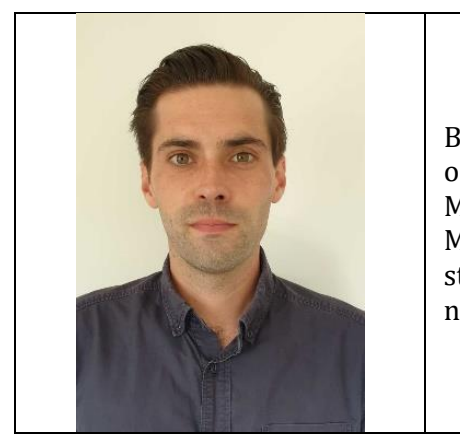

Bastian Muriel was born in Neuilly-sur-Marne, France in 1992. He received is bachelor's degree in organic chemistry from the university of Bordeaux in 2014. In 2016, he joined the LCSO group for his Master thesis, working on the palladium-catalyzed difunctionalization of alkenes. He obtained his Master's degree in 2016 at the University of Bordeaux and then returned to Lausanne for his doctoral studies under the supervision of Prof. Waser. His current research interests lie in the development of new radical-based methodologies exploiting ring strain.

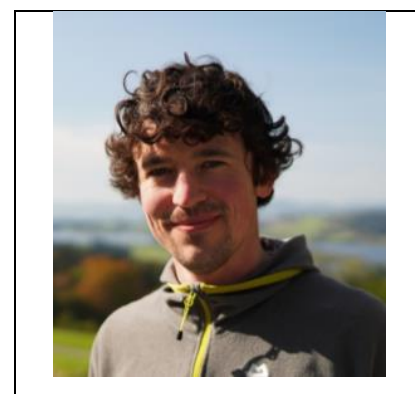

Phillip Greenwood was born in West Sussex, UK in 1991. He received his master's degree from Imperial College London in 2015. He joined the LCSO group in 2015 to carry out his PhD under the supervision of Prof. Jérôme Waser with funding from the SNF and ERC to carry out tethering reactions with palladium catalysis. Graduating from EPFL in 2020, he currently works at Pfizer, Sandwich as a process development chemist. 

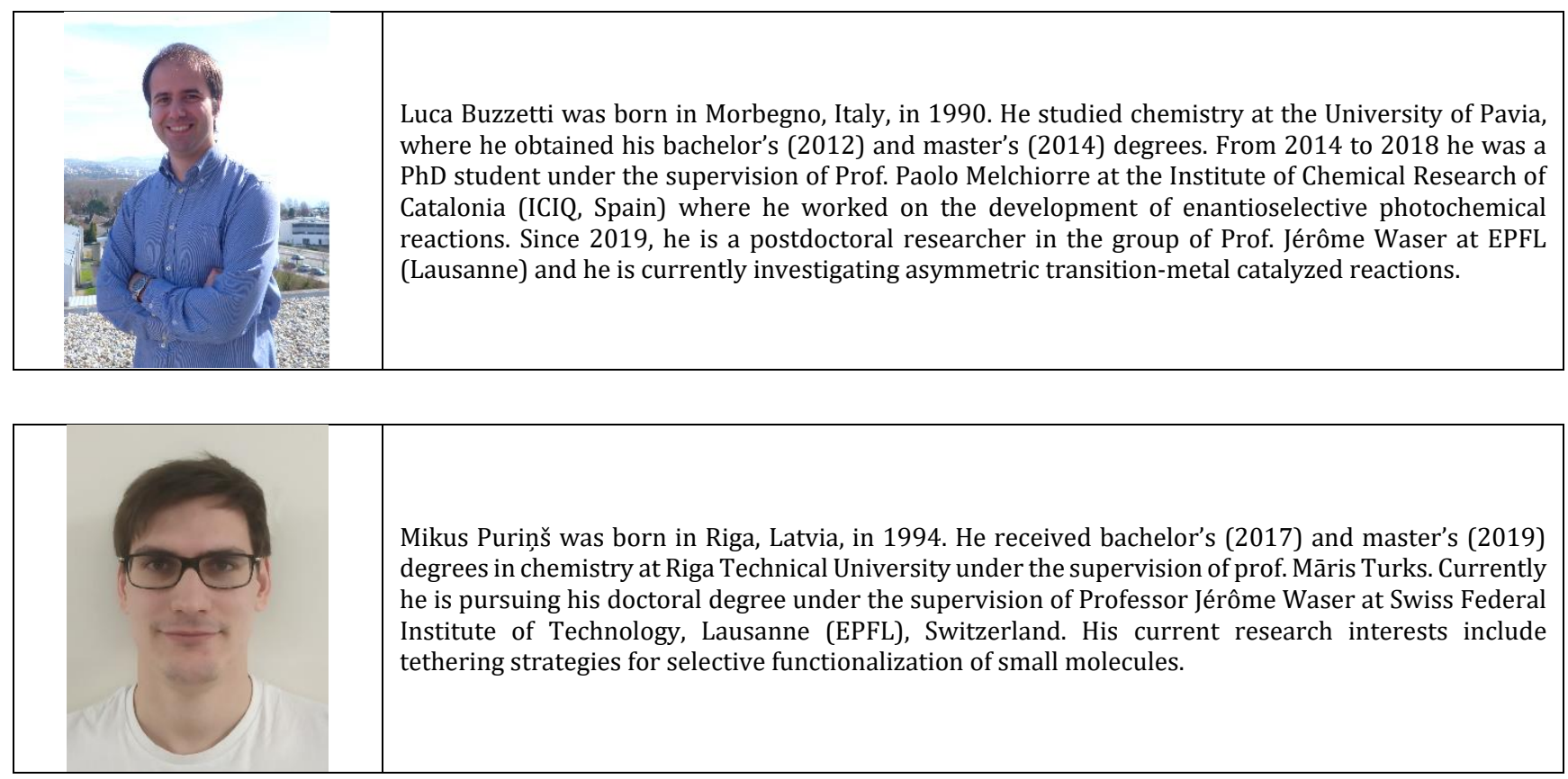